\title{
TIME BOUNDS FOR SHARED OBJECTS IN PARTIALLY SYNCHRONOUS SYSTEMS
}

\author{
A Thesis \\ by \\ JIAQI WANG
}

\begin{abstract}
Submitted to the Office of Graduate Studies of Texas A\&M University in partial fulfillment of the requirements for the degree of

MASTER OF SCIENCE
\end{abstract}

December 2011

Major Subject: Computer Science 


\title{
TIME BOUNDS FOR SHARED OBJECTS IN PARTIALLY SYNCHRONOUS SYSTEMS
}

\author{
A Thesis \\ by \\ JIAQI WANG
}

Submitted to the Office of Graduate Studies of

Texas A\&M University

in partial fulfillment of the requirements for the degree of

\section{MASTER OF SCIENCE}

Approved by:

Chair of Committee, Jennifer L. Welch

Committee Members, Andreas Klappenecker

Alex Sprintson

Head of Department, Duncan M. Walker

December 2011

Major Subject: Computer Science 


\begin{abstract}
Time Bounds for Shared Objects in Partially Synchronous Systems.

(December 2011)

Jiaqi Wang, B.S., Nanjing University

Chair of Advisory Committee: Dr. Jennifer L. Welch
\end{abstract}

Shared objects are a key component in today's large distributed systems. Linearizability is a popular consistency condition for such shared objects which gives the illusion of sequential execution of operations. The time bound of an operation is the worst-case time complexity from the operation invocation to its response. Some time bounds have been proved for certain operations on linearizable shared objects in partially synchronous systems but there are some gaps between time upper bound and lower bound for each operation. In this work, the goal is to narrow or eliminate the gaps and find optimally fast implementations.

To reach this goal, we prove larger lower bounds and show smaller upper bounds (compared to $2 d$ for all operations in previous folklore implementations) by proposing an implementation for a shared object with an arbitrary data type in distributed systems of $n$ processes in which every message delay is bounded within $[d-u, d]$ and the maximum skew between processes' clocks is $\epsilon$.

Considering any operation for which there exist two instances such that individually, each instance is legal but in sequence they are not, we prove a lower bound of $d+\min \{\epsilon, u, d / 3\}$, improving from $d$, and show this bound is tight when $\epsilon<d / 3$ and $\epsilon<u$.

Considering any operation for which there exist $k$ instances such that each instance separately is legal and any sequence of them is legal, but the state of the object is different after different sequences, we prove a lower bound of $(1-1 / k) u$, improving 
from $u / 2$, and show this bound is tight when $k=n$.

A pure mutator only modifies the object but does not return anything about the object. A pure accessor does not modify the object. For a pure mutator $O P_{1}$ and a pure accessor $O P_{2}$, if given a set of instances of $O P_{1}$, the state of the object reflects the order in which the instances occur and an instance of $O P_{2}$ can detect whether an instance of $O P_{1}$ occurs, we prove the sum of the time bound for $O P_{1}$ and $O P_{2}$ is at least $d+\min \{\epsilon, u, d / 3\}$, improving from $d$. The upper bound is $d+2 \epsilon$ from our implementation. 


\section{ACKNOWLEDGMENTS}

I would like to thank my committee chair, Dr. Welch, and my committee members, Dr. Klappenecker, and Dr. Sprintson, for their guidance and support throughout the course of this research. I also want to thank Dr. Lee, for her suggestions and reviews throughout the discussions of this research.

Thanks also go to my friends and colleagues and the department faculty and

staff for making my time at Texas A\&M University a great experience. I also want to extend my gratitude to the NSF, which provided the research funding.

Finally, thanks to my mother and father for their encouragement. 


\section{TABLE OF CONTENTS}

CHAPTER

INTRODUCTION . . . . . . . . . . . . . . . . . . . . . . . 1

A. What Is the Problem . . . . . . . . . . . . . . 1

1. Shared Objects . . . . . . . . . . . . . 1

2. Time Bounds . . . . . . . . . . . . . . . . . 1

3. Problem Statement . . . . . . . . . . . . . 4

B. Previous Related Work . . . . . . . . . . . . . 4

C. What Is Done in the Thesis . . . . . . . . . . 6

II ARBITRARY DATA TYPES . . . . . . . . . . . . . 9

A. Deterministic Object . . . . . . . . . . . . . 9

B. Immediately Non-commuting . . . . . . . . . . . . . . . . 10

C. Eventually Non-commuting and Eventually Non-permuting 13

D. Mutator, Accessor and Overwriter . . . . . . . . . 16

III SYSTEM MODEL . . . . . . . . . . . . . . . 18

A. Overview . . . . . . . . . . . . . . . . . 18

B. Detailed Specifications . . . . . . . . . . . . . . 19

1. Process . . . . . . . . . . . . . . . . 19

2. View of Process . . . . . . . . . . . . . . 20

3. Run ................... . 21

4. Correctness of Algorithm . . . . . . . . . . 23

IV LOWER BOUNDS FOR LINEARIZABLE SHARED OBJECT 25

A. Standard Time Shift . . . . . . . . . . . . . . . 25

B. Modified Time Shift . . . . . . . . . . . . 27

1. Chopping and Extending . . . . . . . . . . . . 29

2. Appending to an Initialized Run . . . . . . . . . . . 31

C. Strongly Immediately Non-self-commuting Operation Types 33

D. Eventually Non-self-last-permuting Operation Types . . . . 40

E. Immediately Non-commuting Pairs of Operation Types . . 46

V UPPER BOUNDS FOR LINEARIZABLE OBJECTS . . . . . . 57

A. Main Idea of the Implementation . . . . . . . . . . 57 
CHAPTER

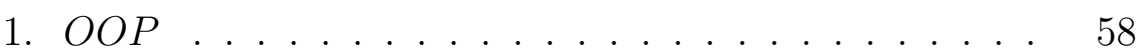

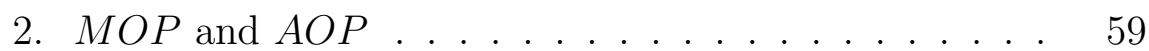

B. Pseudocode .................... 61

C. Correctness ................. . . 61

1. Summary of Observations . . . . . . . . . 61

2. Termination ............... 63

3. Linearizability . . . . . . . . . . . . . . 64

a. Step I . . . . . . . . . . . . . . . . . . 64

b. Step II . . . . . . . . . . . . . . . . . . 65

c. Step III . . . . . . . . . . . . . . . . . . . 70

D. Analysis of Implementations . . . . . . . . . . . . 72

VI TIME BOUNDS FOR SPECIFIC SHARED OBJECTS . . . . . 74

A. Operations on Read/Write/Read-Modify-Write Registers . 74

B. Operations on Queues and Stacks . . . . . . . . . . . 74

C. Operations on Trees . . . . . . . . . . . . . 75

VII CONCLUSION ....................... 77

REFERENCES . . . . . . . . . . . . . . . . . . 79

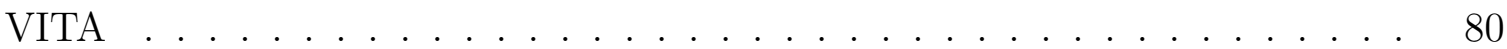




\section{LIST OF TABLES}

TABLE

I Summary of Operation Time Bounds on Read/Write/Read-ModifyWrite Register . . . . . . . . . . . . . . . . . . . . . . 75

II Summary of Operation Time Bounds on Queue . . . . . . . . . . . 75

III Summary of Operation Time Bounds on Stack . . . . . . . . . . 76

IV Conclusions of Operation Time Bounds on Tree . . . . . . . . . 76 


\section{LIST OF FIGURES}

FIGURE

Page

1 Operation Time and Linearizability . . . . . . . . . . . . . . 3

2 Shared Object Implementation in Message Passing System . . . . . . 18

3 Example of Standard Time Shift . . . . . . . . . . . . . . 26

4 Standard Time Shift and Modified Time Shift . . . . . . . . . . . . . 29

$5 \quad$ Chopping and Appending to an Initialized Run . . . . . . . . . . . 31

6 Proof Structure of Theorem C.1 . . . . . . . . . . . . . 35

7 Step 1 in Theorem C.1 . . . . . . . . . . . . . . . 36

8 Step 2 in Theorem C.1 . . . . . . . . . . . . . . . 38

$9 \quad$ Step 3 in Theorem C.1 . . . . . . . . . . . . . . . . . . 39

$10 \quad$ Message Delays in $R_{1}\left(R_{1}^{\prime}\right) \ldots \ldots \ldots$. . . . . . . . . 41

$11 \quad$ Run $R_{1}\left(R_{1}^{\prime}\right) \ldots \ldots \ldots \ldots \ldots$. . . . . . . . . . . . . . . . .

$12 \quad$ Example of Standard Time Shift of $R_{1} \ldots \ldots$. . . . . . . . . . 43

$13 \quad$ Message Delays in $R_{2}\left(R_{2}^{\prime}\right) \ldots \ldots \ldots$. . . . . . . . 44

$14 \quad$ Run $R_{2}\left(R_{2}^{\prime}\right) \ldots \ldots \ldots \ldots \ldots \ldots$

15 Proof Structure of Theorem E.1 . . . . . . . . . . . . . . 50

$16 \quad$ Step 1 in Theorem E.1 . . . . . . . . . . . . . . 52

17 Step 2 in Theorem E.1 . . . . . . . . . . . . . . 53 


\section{CHAPTER I}

\section{INTRODUCTION}

A. What Is the Problem

\section{Shared Objects}

Shared objects are a key component in today's large distributed systems. Applications ranging from electronic commerce to social media on hand-held devices require shared data. These applications provide the functions of sharing, updating and also accessing information (stored in shared objects) in message passing systems. These functions are realized via operations on the shared object. Each operation has an invocation and a response. Different from the object which can be accessed by only one process, since the operations on the shared object are executed by multiple processes, the operations can be executed simultaneously. In fact, the executions of operations on a shared object overlap in time. Each object has a sequential specification which determines the legal operations and the legal operation sequences on the object. For each shared object, linearizability defines a desired behavior of operations (including the operations which overlap in time), which follows the sequential specification of the object. We will give more detailed explanation and formal definition for linearizability in the following chapters.

\section{Time Bounds}

In the applications, the sooner an operation responds, the earlier the application can execute the next step. So we want each operation to respond as fast as possible. Time bound for an operation is the worst-case time complexity of this operation.

This thesis follows the style of IEEE Transactions on Computers. 
The time upper bound for an operation is the minimum value of the worst-case time complexity of this operation in all the existing correct implementations. The time lower bound for an operation is the minimum value of the worst-case time complexity of this operation in all the possible correct implementations. It means in all the correct implementations, for each operation, the worst-case time complexity must reach the lower bound, otherwise the implementation cannot be correct. So if the upper bound equals the lower bound, it means the lower bound is a tight bound and we have found the fastest implementation for this operation; if there exists a gap between the upper bound and lower bound for an operation, either there exists some faster implementation which hasn't been found, or the lower bound is not a tight bound.

Now we give a simple example to show why the implementation must be incorrect if operations respond faster than their lower bounds. This example is shown on a shared read-write register. Write operation is expressed by write(arg) where arg is the value to be written into the register. Read operation is expressed by read(ret) where ret is the return value of the read operation. In Fig. 1(a), the read operation is invoked after the two write operations complete. By linearizability, the read operation should return 1 . However, due to the message delay from process $p_{i}$ to $p_{j}$, the read operation responds before $p_{j}$ learns the existence of write(1), so the read operation returns 0 , violating the linearizability of the register. If we have a longer operation, the problem can be solved. As Fig. 1(b) depicts, if the execution of write(1) is longer such that it overlaps with read $(0)$, then write $(0) \circ \operatorname{read}(0) \circ$ write $(1)$ is a legal permutation and it respects the real time sequence. As Fig. 1(c) depicts, if the execution of the read operation is longer, such that $p_{j}$ learns the existence of write $(1)$ before the read operation responds, then the read operation can return 1 after learning the existence of write(1). 


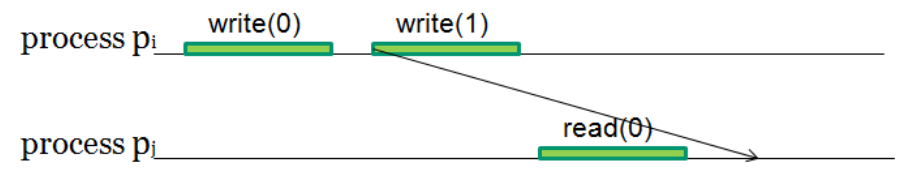

(a) Incorrect Implementation

process $\mathrm{p}_{\mathrm{i} \_ \text {write }(0) \quad \text { write }(1)}$

(b) Longer Write Operation

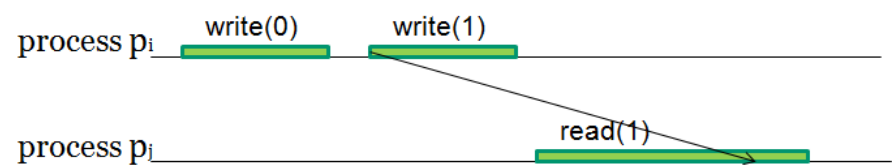

(c) Longer Read Operation

Fig. 1. Operation Time and Linearizability 


\section{Problem Statement}

From the view of the application, we want the operations to respond faster. But to ensure linearizability of the shared object, the operations cannot respond too fast. So the questions become: without violating the linearizability of the object, how fast can the operations on a shared object respond? And how can one make them respond quickly?

To guarantee the linearizability of an object of arbitrary data type, a centralized mechanism can perform each operation with time at most $2 d$ in the worst case if the system provides message delay bound $d$, since the message from the invoking process to the control center takes at most $d$ and the response message also takes at most $d$. Alternatively, one can use a total order broadcast primitive, but this is not faster than the centralized scheme when taking into account the time overhead to

implement the totally ordered broadcast on top of a point-to-point message system [1]. Increasing pressure to speed up applications raises the question whether operations can be executed faster than $2 d$.

\section{B. Previous Related Work}

Lipton and Sandberg [5] started to describe fundamental performance limitations for distributed systems that provide sequential consistency (a consistency condition weaker than linearizability). Then Attiya and Welch [1] extended Liption and Sandberg's work to show separation w.r.t. time complexity of operations between sequential consistency and linearizability. They also considered shared objects of type other than read/write registers - specifically, stacks and queues.

To ensure serializability of transactions, Weihl [8] proposed two algorithms using conflict relations based on the commutativity of operations. The two algorithms 
permit the results returned by operations to be used in determining conflicts, thus permitting higher levels of concurrency than is otherwise possible.

Inspired by Weihl's work, Kosa [3] proved tight bounds for distributed implementations of different operations types based on the commutativity relationships between operations. Those operations are classified based on their characteristic properties. Kosa [3] used algebraic approaches to specify the properties of operations and defined the operation types such as immediately non-self-commuting operations, immediately self-commuting operations, pure mutators and accessors (definitions of them are given in Chapter II). Then Kosa focused on immediately non-self-commuting operations or pairs of operations which immediately do not commute with each other in a perfectly synchronous system and proved tight time bounds. What's more, Kosa extended the time lower bound proof from a pair of operations to a commutativity graph.

We also study immediately non-self-commuting operations and pairs of operations which immediately do not commute with each other. But we prove the time bounds in a partially synchronous system where the message delay and local clock skew are constrained by certain bounds. And we show that if the local clock skew is smaller than one third of the message delay upper bound, the time bound we proved for immediately non-self-commuting operations is tight. Immediately non-selfcommuting operations include read-modify-write on a register, dequeue on a queue and pop on a stack.

Kosa also extended some time lower bounds in partially synchronous systems from previous lower bounds of read and write operations [1]. However the previous time lower bound for the eventually non-self-commuting operations and pure accessors left gaps with respect to their upper bounds. We extend the number of concurrent

In a perfectly synchronous system, every process has the same local time. 
operations from 2 to an arbitrary positive number $k$, which means we study permutations of $k$ operations instead of the commutativity of two operations. Then we prove a tight time bound which can be applied to eventually non-self-permuting operations such as write on a register, enqueue on a queue and push on a stack.

There are also some results on pairs of operations in partially synchronous system. Mavronicolas and Roth [7] proved for write and read operations on a shared object, the time lower bound for the sum of them is at least $d+\min \{\epsilon, u\} / 2$. However, this result only applies for a certain class of algorithms with some constraint. In this work, we remove the constraint. Also, a more general result instead of only for write and read operations is obtained. In this study, it is found that the time bound for a pair of operations is impacted by the properties of individual operations, such as eventually self-commuting, eventually non-self-commuting, and self-overwriting.

\section{What Is Done in the Thesis}

This work studies time bounds for linearizable implementations of arbitrary shared objects, with applications to some commonly used shared objects, such as read/write registers, queues, stacks and trees. Each shared object provides a specific semantic structure and is equipped with a specific set of operations. Kosa [3] characterized operations by axioms on what operation sequences are "legal" and proved a variety of upper and lower bounds in different models. But there still exist gaps between the lower and upper bounds for many commonly used data types. In this work, Kosa's approach is extended as follows:

- We derive an algorithm that exploits the axiomatic properties of different operations to reduce the running time of each operation to less than $2 d$.

- We obtain better lower bounds on the time complexity of certain types of op- 
erations, by exploiting the message delay uncertainty and the presence of more than 2 processes in the system.

- As a result of the new upper and lower bounds, the gap is reduced and in some cases we have tight bounds.

- New classifications of operations are also considered based on new properties relevant to the lower bounds.

Extending Kosa's work, we consider the following properties of operations:

- whether the operation modifies the object;

- whether the operation returns information about the object;

- whether the operation commutes with itself; and

- whether the operation overwrites the whole state of the object.

For example, suppose on a shared register $x$, there are three operations: read, write and increment.

1. The read operation does not modify the value of $x$ and it returns the value. Besides, the read operation commutes with itself, because in an operation sequence, two consecutive read operations $r_{1}, r_{2}$ return the same value and if we exchange the positions of $r_{1}$ and $r_{2}$ in an operation sequence, the new operation sequence is still "legal".

2. The write operation modifies the value of $x$ but it does not return any information about $x$. Besides, the write operation does not eventually commute with itself (refer to Definition C.3 in Chapter II), because if we exchange the positions of two different and consecutive write operations in an operation sequence, 
the value of $x$ after the two writes will be different from that before position exchanging. What's more, the write operation overwrites the whole state of $x$, because whatever the previous operation history is, the latest write operation determines the value of $x$.

3. In contrast, although the increment operation also modifies $x$, it commutes with itself and it doesn't overwrite the whole state of $x$.

Finally we summarize the time bounds on the typical shared object types register, queue, stack and tree. On these object types there are operations which satisfy the properties we work on. So we can apply lower and upper time bounds on these objects easily. 


\section{CHAPTER II}

\section{ARBITRARY DATA TYPES}

The data type of an object specifies a set of operations which are meaningful to this type. Each operation is composed of an invocation and a response, and the meaning of the operation can be reflected by modifications on the object and the response of the operation. In this work we only consider deterministic objects.

\section{A. Deterministic Object}

In this chapter, Chapter III and Chapter IV, we use $O$ to specify an instance of an object, use $O P$ to specify an operation type, such as write operation on a register, and use $o p$ for an instance of this operation type. We write $o p \in O P$ to express that $o p$ is an operation of type $O P$. We also use $O P(\arg , \mathrm{ret})$ to denote an operation $o p$ of type $O P$ which takes arg as the operation argument and takes ret as the return value, so op $=O P(\arg , r e t)$. In Chapter $\mathrm{V}$, we use op(arg) as an operation invocation with argument $\arg$.

For an object which is accessed by a single process, since all the operations on this object are executed sequentially, there is a sequential specification to determine the desired behavior of this object. For each object, the sequential specification contains a set of operations and a set of sequences of operations. The set of sequences of operations contains all the legal operation sequences. This set defines whether an operation sequence is legal. For example:

- In the sequential specification of a read-write register, the set of legal sequences of operations contains all the operation sequences in which each read operation returns what the latest write operation before it writes into the register. 
- In the sequential specification of a queue, in each legal operation sequence, the dequeue operation removes and returns the head of the queue.

- In the sequential specification of a stack, in each legal operation sequence, the pop operation removes and returns the element at top of the stack.

Definition A.1 A deterministic object satisfies the following condition:

For every operation sequence $\rho$ and every operation type $O P$, if both $\rho \circ O P($ arg, ret $)$ and $\rho \circ O P\left(\arg , r^{\prime} t^{\prime}\right)$ are legal, then ret $=$ ret $^{\prime}$.

In the whole work, we only consider deterministic objects.

B. Immediately Non-commuting

Definition B.1 Immediately non-commuting - For any two operation types $O P_{1}$ and $O P_{2}$, if there exist an operation sequence $\rho$, operation $o p_{1} \in O P_{1}$, and operation $o p_{2} \in O P_{2}$, such that $\rho \circ o p_{1}$ and $\rho \circ o p_{2}$ are legal but at least one of the below is illegal:

(1) $\alpha=\rho \circ o p_{1} \circ o p_{2}$

(2) $\beta=\rho \circ o p_{2} \circ o p_{1}$

we say $O P_{1}$ and $O P_{2}$ are immediately non-commuting.

For example, suppose $O P_{1}$ is the read operation and $O P_{2}$ is the write operation on a shared register. If $\rho=$ write $(0)$, then $\rho \circ$ write $(1), \rho \circ \operatorname{read}(0)$ and $\rho \circ \operatorname{read}(0) \circ$ write $(1)$ are all legal, but $\rho \circ$ write $(1) \circ \operatorname{read}(0)$ is not legal. Thus, the read and write operation are immediately non-commuting operations.

Definition B.2 Immediately non-self-commuting - In the definition of immediately non-commuting, if $O P_{1}=O P_{2}$, then $O P_{1}$ is immediately non-self-commuting. 
Notes: in the following chapters we describe two operation types which are not immediately non-commuting by immediately commuting and describe an operation type which is not immediately non-self-commuting by immediately self-commuting.

Definition B.3 Strongly Immediately non-self-commuting - In the definition of immediately non-commuting, if both $\alpha$ and $\beta$ are illegal and $O P_{1}=O P_{2}$, then $O P_{1}$ is strongly immediately non-self-commuting.

Many common immediately non-self-commuting operation types are also strongly immediately non-self-commuting operation types. First we show three commonly used operation types which are both immediately non-self-commuting and also strongly immediately non-self-commuting.

- Read-Modify-Write on a register $R$ : Suppose $\rho=$ write $(0), o p_{1}$ reads the value of $R$ and writes 1 into $R$ and $o p_{2}$ reads the value of $R$ and writes 2 into $R$. Because both $\rho \circ o p_{1}$ and $\rho \circ o p_{2}$ are legal, both $o p_{1}$ and $o p_{2}$ should return 0 . But it is illegal if $o p_{2}$ returns 0 in $\rho \circ o p_{1} \circ o p_{2}$ and it is illegal if $o p_{1}$ returns 0 in $\rho \circ o p_{2} \circ o p_{1}$.

- Pop on a stack $S$ : Suppose after $\rho, S$ has one element $X$. $o p_{1}$ and $o p_{2}$ pop the top of $S$ and return the element they pop. Because $\rho \circ o p_{1}$ and $\rho \circ o p_{2}$ are legal, both $o p_{1}$ and $o p_{2}$ should return $X$. Then both $\rho \circ o p_{1} \circ o p_{2}$ and $\rho \circ o p_{2} \circ o p_{1}$ are illegal, because $o p_{2}$ should return nothing in $\rho \circ o p_{1} \circ o p_{2}$ and $o p_{1}$ should return nothing in $\rho \circ o p_{2} \circ o p_{1}$.

- Dequeue on a queue $Q$ : Suppose after $\rho, Q$ has one element $X$. $o p_{1}$ and $o p_{2}$ are both dequeue operations which remove the head of $Q$ and return the element they remove. Because $\rho \circ o p_{1}$ and $\rho \circ o p_{2}$ are legal, both $o p_{1}$ and $o p_{2}$ return $X$. 
Then both $\rho \circ o p_{1} \circ o p_{2}$ and $\rho \circ o p_{2} \circ o p_{1}$ are illegal, because $o p_{2}$ returns nothing if $\rho \circ o p_{1} \circ o p_{2}$ is legal and $o p_{1}$ returns nothing if $\rho \circ o p_{2} \circ o p_{1}$ is legal.

Next we describe an operation type $\operatorname{UpdateNext}(i, a, b)$ on an integer array of size 2, which is immediately non-self-commuting but not strongly immediately nonself-commuting. UpdateNext $(i, a, b)$ returns the $i$ th element in the array ( $a$ shows the return value) and updates the $(i+1)$ th element with value $b$; and if the $i$ th element is the last one in the array, it modifies nothing.

To show it is immediately non-self-commuting, suppose the array is initialized with $[x, y]$ and previous operation sequence $\rho$ is empty. $o p_{1}=O P(1, x, z)$ where $z \neq y, o p_{2}=O P(2, y, z)$. Then $\rho \circ o p_{1}, \rho \circ o p_{2}$ and $\rho \circ o p_{2} \circ o p_{1}$ are legal. But $\rho \circ o p_{1} \circ o p_{2}$ is illegal.

Now we show $U p d a t e N \operatorname{ext}(i, a, b)$ is not a strongly immediately non-self-commuting operation type. Suppose in contradiction we can find an operation sequence $\rho$, $o p_{1}=O P(i, a, b), o p_{2}=O P\left(j, a^{\prime}, b^{\prime}\right)$ such that both $\rho \circ o p_{1} \circ o p_{2}$ and $\rho \circ o p_{2} \circ o p_{1}$ are illegal. Without loss of generality, we can assume after $\rho$, the array value is $[x, y]$. We show that in all the cases below, there exists some contradiction:

- Case 1: $i=j=1$. Because $\rho \circ o p_{1}$ and $\rho \circ o p_{2}$ are legal, $a=a^{\prime}=x$. Then both $\rho \circ o p_{1} \circ o p_{2}$ and $\rho \circ o p_{2} \circ o p_{1}$ are legal, because no operation changes the first element.

- Case 2: $i=j=2$. Similar to case 1, both $\rho \circ o p_{1} \circ o p_{2}$ and $\rho \circ o p_{2} \circ o p_{1}$ are legal.

- Case 3: $i=1, j=2$. Because $\rho \circ o p_{1}$ and $\rho \circ o p_{2}$ are legal, $a=x$ and $a^{\prime}=y$. Then $\rho \circ o p_{2} \circ o p_{1}$ must be legal, because $o p_{2}$ modifies nothing.

- Case 4: $i=2, j=1$. Similar to case 3, $\rho \circ o p_{1} \circ o p_{2}$ is legal. 
C. Eventually Non-commuting and Eventually Non-permuting

In contrast with immediately non-self-commuting operation types, there exist eventually non-self-commuting operation types. Before we give the definition for them, let's review Kosa [3]'s definitions for "look like" and "equivalent" first.

Definition C.1 Look like - An operation sequence $\rho_{1}$ looks like another operation sequence $\rho_{2}$, if for any operation sequence $\rho_{3}$ where $\rho_{1} \circ \rho_{3}$ is legal, $\rho_{2} \circ \rho_{3}$ is legal.

Definition C.2 Equivalent - Two operation sequences $\rho_{1}$ and $\rho_{2}$ are equivalent if and only if $\rho_{1}$ looks like $\rho_{2}$ and $\rho_{2}$ looks like $\rho_{1}$.

Definition C.3 Eventually non-self-commuting - For any eventually non-selfcommuting operation type $O P$, there exists an operation sequence $\rho$, and $o p_{1}, o p_{2} \in$ $O P$, such that $\rho \circ o p_{1}$ and $\rho \circ o p_{2}$ are legal, and $\rho \circ o p_{1} \circ o p_{2}$ and $\rho \circ o p_{2} \circ o p_{1}$ are not equivalent.

An example is the write operation on a read/write register. Suppose after $\rho=$ write $(0)$. $o p_{1}$ writes 1 into the register and $o p_{2}$ writes 2 into the register. After $\rho \circ o p_{1} \circ o p_{2}$, the value of the register is 2 . After $\rho \circ o p_{2} \circ o p_{1}$, the value of the register is 1. Therefore $\rho \circ o p_{1} \circ o p_{2}$ does not look like $\rho \circ o p_{2} \circ o p_{1}$ because there exists an operation $\operatorname{read}(1)$ such that $\rho \circ o p_{2} \circ o p_{1} \circ \operatorname{read}(1)$ is legal while $\rho \circ o p_{1} \circ o p_{2} \circ \operatorname{read}(1)$ is illegal.

Now we have defined immediately non-commuting and eventually non-commuting. We can see that both of them are defined based on the order of 2 operations. However, if we want to implement a shared object in a message passing system, the message passing system usually has more than 2 processes and it is reasonable that more operations are invoked concurrently. Thus we consider the order of $n(n \geq 2)$ overlapping 
operations. We call an operation sequence composed of the $n$ operations a permutation of the $n$ operations. So we use permuting to describe relationships between the $n$ operations. Similar to eventually non-self-commuting, the property of eventually non-self-permuting is determined by if two different permutations of $n(n \geq 2)$ operations are equivalent or not.

Before we give the definition of non-self-permuting, we continue comparing commuting and permuting. If there are only 2 operations, there are only 2 permutations of operation sequence, depending on which operation is the first one. So either the two permutations are equivalent or not equivalent. If there are $n$ operations where $n>2$, there are $n$ ! permutations, which means in the $n$ ! permutations, some permutations may be equivalent while some other permutations may be different. An extreme case is that any two different permutations of the $n$ operations are not equivalent. On the opposite side, another extreme case is that all the different permutations are equivalent. So between the strong non-permuting (the first extreme case) and permuting (the second extreme case), there might be different levels of the non-permuting properties.

Now let's start the definitions related to the non-permuting property.

Definition C.4 Eventually non-self-any-permuting - An operation type OP is eventually non-self-any-permuting if and only if there exist an operation sequence $\rho$, and $o p_{1} \in O P, o p_{2} \in O P, \ldots o p_{n} \in O P$ for any $n>1$, such that:

1. $\rho \circ o p_{i}(0<i \leq n)$ is legal;

2. there exist at least two legal permutations of the $n$ operations; and

3. any two different legal permutations of the $n$ operations are not equivalent.

Definition C.5 Eventually non-self-last-permuting - An operation type OP is 
eventually non-self-last-permuting if and only if there exist an operation sequence $\rho$, and $o p_{1} \in O P, o p_{2} \in O P, \ldots o p_{n} \in O P$ for any $n>1$, such that:

1. $\rho \circ o p_{i}(0<i \leq n)$ is legal;

2. there exist at least two legal permutations of the $n$ operations; and

3. any two different legal permutations $\pi, \pi^{\prime}$ of the $n$ operations are not equivalent if $\operatorname{last}(\pi) \neq \operatorname{last}\left(\pi^{\prime}\right)(\operatorname{last}(\pi)$ is the last operation in $\pi)$.

Of course the eventually non-self-any-permuting operations are eventually nonself-last-permuting operations, because two different permutations with different last operations belong to two different permutations. Let's see some examples below:

- Write: The write operation on a read/write register is an eventually non-selflast-permuting operation but not an eventually non-self-any-permuting operation. Because if $\operatorname{last}(\pi)=\operatorname{last}\left(\pi^{\prime}\right)$, then $\rho \circ \pi$ and $\rho \circ \pi^{\prime}$ are equivalent. If $\operatorname{last}(\pi) \neq \operatorname{last}\left(\pi^{\prime}\right)$, they are not equivalent. So for any two permutations, as long as the last operation is the same, they are equivalent, which means write operation is not eventually non-self-any-permuting.

- Push: The push operation on a stack is eventually non-self-any-permuting operations. Suppose the stack is initialized with an empty stack, $\rho$ is empty, and let $o p_{i}$ push the integer $i$ onto the stack. For any two different permutations $\pi$ and $\pi^{\prime}, \rho \circ \pi$ and $\rho \circ \pi^{\prime}$ are not equivalent because a sequence of continuous pop operations can distinguish the differences between the two permutations.

- Enqueue: The enqueue operation on a queue is eventually non-self-any-permuting operations. Suppose the queue is initialized with an empty queue, $\rho$ is empty, and let $o p_{i}$ enqueues the integer $i$ into the queue. For any two different permutation $\pi$ and $\pi, \rho \circ \pi$ and $\rho \circ \pi^{\prime}$ are not equivalent because a sequence of 
continuous dequeue operations can distinguish the differences between the two permutations.

Definition C.6 Eventually self-commuting - An operation type OP is eventually self-commuting if and only if for any operation sequence $\rho$, and any $o p_{1}, o p_{2} \in O P$ such that $\rho \circ o p_{1}$ and $\rho \circ o p_{2}$ are legal, $\rho \circ o p_{1} \circ o p_{2}$ and $\rho \circ o p_{2} \circ o p_{1}$ are legal and equivalent.

For example, consider the insert and delete operations on a set. The order of insertion or deletion does not affect the elements in the set, so they are eventually self-commuting.

D. Mutator, Accessor and Overwriter

Definition D.1 Mutator - An operation which modifies the object. For any mutator $O P$, there exist an operation sequence $\rho$ and operation $o p_{1} \in O P$, such that $\rho \circ o p_{1}$ and $\rho$ are not equivalent.

Definition D.2 Accessor - An operation which return some information about the object. For any accessor $O P$, there exist a legal operation sequence $\rho$ and operation instance op $\in O P$, such that $\rho \circ$ op is illegal.

Definition D.3 Pure Mutator - Mutator which is not accessor.

Definition D.4 Pure Accessor - Accessor which is not mutator.

Definition D.5 Non-overwriter - A mutator OP is a non-overwriter if there exist operation sequences $\rho$, and $o p_{1}, o p_{2} \in O P$ such that $\rho \circ o p_{1} \circ o p_{2}$ and $\rho \circ o p_{2}$ are not equivalent. 
For example, suppose $o p_{1}$ and $o p_{2}$ are increment operations which return nothing on a shared register and $o p_{3}$ is the read operation on this register. Suppose $\rho=$ write $(0), o p_{1}$ increases the value by $1, o p_{2}$ increases the value by 2 and $o p_{3}$ is a read operation. Then if $\rho \circ o p_{1} \circ o p_{2} \circ o p_{3}$ is legal, $o p_{3}$ should return 3 and if $\rho \circ o p_{2} \circ o p_{3}$ is legal, then $o p_{3}$ should return 2. Therefore, one of them is illegal and increment is a non-overwriter. 


\section{CHAPTER III}

\section{SYSTEM MODEL}

\section{A. Overview}

The shared object system contains three layers: (1) application layer; (2) object implementation layer; and (3) message passing layer. Each layer is built on another layer as shown in Fig. 2:

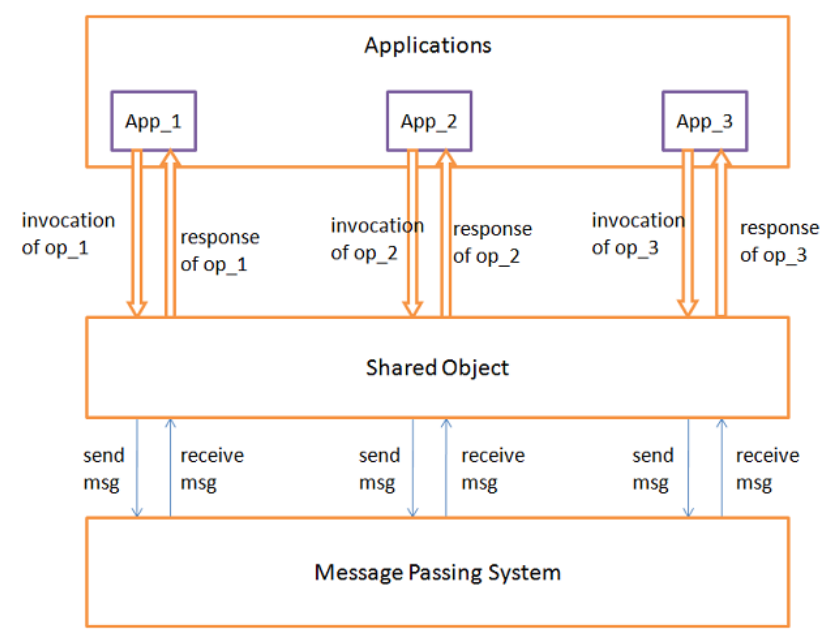

Fig. 2. Shared Object Implementation in Message Passing System

The top layer is the application layer, which sends operation invocations to the middle layer and receives the operation responses from the middle layer. Each operation must be invoked and respond in the same process. Each process can have at most one pending operation, which means only after returning the response of the previous operation, a process can invoke a new operation. Besides, in a finite time period, every process can invoke only a finite number of operations.

The middle layer is the implementation of the shared object, which takes oper- 
ation invocations from the application layer as input and takes operation responses as output. It sends and receives messages via the base layer - the message passing system.

The base layer is the message passing system, which takes the message sending events as input and takes message receiving events as output. The message passing system provides communication between processes via reliable message deliveries, which can guarantee: a) every received message must have been sent by some process before; b) every message is received by the destination process eventually; c) every message is received by the destination process only once.

B. Detailed Specifications

\section{Process}

Each process is modeled as a state machine with a set of states and a transition function. The state set has a subset of initial states. The transition function takes as inputs:

- current state

- an input event (operation invocation or message receipt or timer going off), and

- clock time

and produces as outputs:

- new state and

- set of output events (at most one operation response, and for each other process, at most one message to be sent). 
Timers are part of the state: each timer is a special kind of variable that holds either $\perp$ or a real number that indicates the clock time when the process should take a step. If the input event is a timer going off, then there must be at least one timer in the current state that is equal to the clock time. In the new state, each timer must be set either to $\perp$ or to some value that is larger than the clock time.

A state is quiescent if no timer is set. Thus the process will not do anything until it receives a message or gets an operation invocation.

A step of a process is a quintuple <current state, input event, clock time, new state, set of output events $>$, which captures the application of the transition function. We denote the clock time of step $\sigma_{i}$ by $\operatorname{clock} \_t i m e\left(\sigma_{i}\right)$.

\section{View of Process}

A view of process $p_{j}$ is a (finite or infinite) sequence $\sigma_{1}, \sigma_{2}, \ldots$ of steps of $p_{j}$ such that

- in $\sigma_{1}$, the current state is quiescent;

- the current state in $\sigma_{i}$ equals the new state in $\sigma_{i-1}$ for all $i>1$;

- if $\sigma_{i}$ includes an operation invocation, then no operation is currently pending at $p_{j}$ (the constraint on the application);

- the clock times are increasing, and for any finite number $x$, there are only a finite number of steps whose clock times are less than $x$; and

- the clock time of $\sigma_{i}$ is less than or equal to every timer in the current state for all $i$.

A complete view is a view which either is infinite or ends in a quiescent state. As a result of the last two conditions for a view, if a timer remains set, then a step must 
happen at that clock time. For modeling convenience, we assume each message sent has a unique id that also indicates the sender and the recipient.

A timed view of process $p_{j}$ is a view of $p_{j}$ together with a nonnegative real number $t_{i}$ associated with each step $\sigma_{i}$ in the view. This is the real time at which the step $\sigma_{i}$ occurs and is denoted by real_time $\left(\sigma_{i}\right)$. The following constraint must hold: There exists a constant $c_{j}$ such that, for all $i$, clock_time $\left(\sigma_{i}\right)=$ real_time $\left(\sigma_{i}\right)+c_{j}$. That is, we are only considering clocks that run at the same rate as real time. We call this transition function from real time to clock time the clock function for $p_{j}$.

$\operatorname{shift}(V, x)$ is a shifted timed view, where $V$ is a timed view and $x$ is a real number, by which the real time of each step in $V$ is added.

Claim B.1 If $V$ is a timed view and $x$ is a real number, then shift $(V, x)$ is a timed view.

A timed view $V_{2}$ is an extension of another timed view $V_{1}$ if $V_{1}$ is a prefix of $V_{2}$.

\section{Run}

A run is a set of timed views, one for each process in the set $\Pi$ of $n$ processes, such that every message received in the run is sent exactly once in the run. Note here a message sent is not necessarily received in the run. A complete run is a run in which every timed view in the run is complete and every message is delivered. A finite run is a run in which every timed view is finite.

A run $R$ is admissible if:

- every message received in $R$ is sent $\Delta t(d-u \leq \Delta t \leq d)$ real time earlier in $R$;

- for each message $m$ which is sent at real time $t$ in $R$, but not received in $R$, the view of $m$ 's recipient in $R$ ends before real time $t+d$; and 
- for any two processes $p_{j}$ and $p_{k},\left|c_{j}-c_{k}\right| \leq \epsilon$ (recall that $p_{j}$ 's clock is offset from real time by $c_{j}$ and $p_{k}$ 's clock is offset from real time by $c_{k}$ ) for fixed $\epsilon$ (i.e., the clock skew is bounded).

A run $R_{2}$ is an extension of run $R_{1}$ if, for each process $p_{i}$ in $\Pi$, the timed view for $p_{i}$ in $R_{2}$ is an extension of the timed view for $p_{i}$ in $R_{1}$.

Claim B.2 If $R$ is a finite admissible run, then there exists a complete admissible run that is an extension of $R$.

Given a run $R=\left\{V_{i}: p_{i} \in \Pi\right\}$, where each $V_{i}$ is a timed view of $p_{i}$, and a vector $\vec{x}$ of $n$ real numbers, then $\operatorname{shift}(R, \vec{x})=\left\{\operatorname{shift}\left(V_{i}, x_{i}\right): p_{i} \in \Pi\right\}$.

Claim B.3 If $R$ is a run and $\vec{x}$ is a vector of real numbers, then shift $(R, \vec{x})$ is a run. Furthermore, the shift operator preserves the property of being complete and the property of being finite, but does not necessarily preserve the property of being admissible.

A run $R_{2}$ can be appended to another run $R_{1}$ by appending each timed view in $R_{2}$ to the corresponding timed view in $R_{1}$. We say $R_{2}$ is appendable to $R_{1}$ if:

1. $R_{1}$ ends with every process being quiescent (so each timed view is finite); and

2. for each process

- last state in its timed view in $R_{1}$ equals the first state in its timed view in $R_{2}$

- the first clock time in its timed view in $R_{2}$ is greater than the last clock time in its timed view in $R_{1}$, and

- the clock function for the process is the same in $R_{1}$ and $R_{2}$. 
Claim B.4 Based on the definition of a run, the result of this appending operation is a run.

A run is initialized if processes start in initial states corresponding to the initial value of the object.

\section{Correctness of Algorithm}

For a shared object, since operation executions overlap in time, there must be some rule to specify the desired behavior or correctness for the overlapping operation. Linearizability [2] (or atomicity [4]) is a popular and easy-to-use consistency condition for such shared objects which gives the illusion of sequential execution of operations.

Linearizability: There exists a permutation $\pi$ of all the operations in each complete admissible run $R$ such that:

- a) for each object $O$, the restriction of $\pi$ to operations on the object $O$ is legal;

- b) if the response of $o p_{1}$ occurs before the invocation of $o p_{2}$ in $R$, then $o p_{1}$ appears before $o p_{2}$ in $\pi$.

The first criteria follows the sequential specification to determine if the operation sequence $\pi$ is legal. The second one respects the real time sequence. Two operations that overlap in $R$ may appear in $\pi$ in either order, but for non-overlapping operations, the operation which completes earlier must appear in $\pi$ before the one which is invoked later.

A distributed algorithm is correct if, in every complete admissible run $\mathrm{R}$ of the algorithm:

- Every operation invocation has a matching response, and every response has a matching invocation (i.e., every operation completes and invocations and responses are properly interleaved). 
- Linearizbility of the object is not violated.

We make the following three assumptions about the algorithms we consider, somewhat restricting the class of algorithms for which our lower bounds apply.

1. Bounded-Time Operations: There exists a bound $B_{o p}$ such that every operation invoked in every complete admissible run has its response within $B_{o p}$ time.

2. Bounded Quiescence: There exists a bound $B_{q}$ such that for every complete admissible run containing a finite number of operation invocations, the run is finite and every process is quiescent within $B_{q}$ real time after the last operation response occurs in the run. Besides, each process remains quiescent until a new operation is invoked or a new message is received.

3. History-Obliviousness: Let $R_{1}$ and $R_{2}$ be two complete admissible runs such that for some process $p_{i}$, the same finite sequence of operations is executed by $p_{i}$ in both $R_{1}$ and $R_{2}$, and the other processes do not execute any operations in $R_{1}$ or $R_{2}$. Because of bounded-time operations and bounded quiescence, $R_{1}$ and $R_{2}$ are both finite. Then for each process $p_{j}$, the final state of $p_{j}$ is the same in both $R_{1}$ and $R_{2}$. I.e., nothing about the clock times at which events occurred, or the order in which messages arrived is recorded permanently in the states; all that matters is the sequence of operations executed. 


\section{CHAPTER IV}

\section{LOWER BOUNDS FOR LINEARIZABLE SHARED OBJECT}

Three factors may impact the time bound of operations: message delay upper bound $d$, message delay uncertainty $u$ and local clock skew bound $\epsilon$. We already know the optimal $\epsilon$ is smaller than $u$.

In this chapter, we denote the maximum of the worst-case time from the invocation of each operation $o p \in O P$ to the response of $o p$ in the fastest implementation by $|O P|$.

Before we analyze the time lower bound of operations, we describe a broadly used technique - standard time shift and introduce a new technique - modified time shift, which helps us prove larger lower bounds.

\section{A. Standard Time Shift}

We consider a run in which messages between every two processes $p_{i}$ and $p_{j}$ have fixed time delay, denoted by $d_{i, j}$. In the standard time shift, the local clock of each process $p_{i}$ is shifted by certain amount clock_shift $(i)$. Then in the run resulting from the standard shift, the new message delay $d_{i, j}^{\prime}$ is [6]:

$$
d_{i, j}^{\prime}=d_{i, j}-\operatorname{clock} \_s h i f t(i)+\text { clock_shift }(j)
$$

With the new message delays, each message is still received at the same local time, which means no process notices any difference from before the standard time shift. Therefore the executions of operations are exactly the same as before the standard time shift.

If the original delays and the shift amounts are such that the old and new delays are both admissible, then certain deductions can be made to show some lower bounds. 


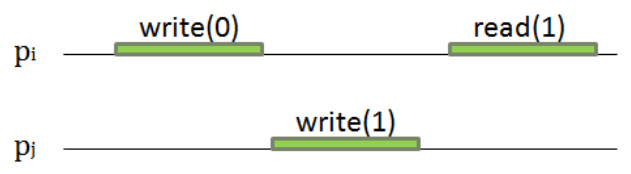

(a) Before Time Shift

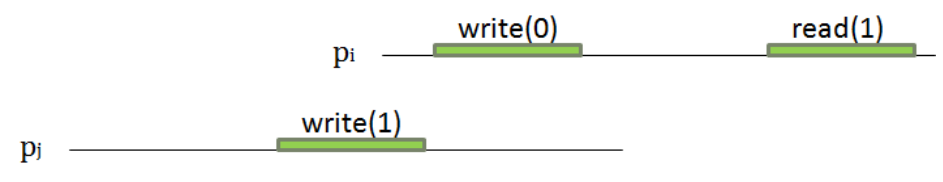

(b) After Time Shift

Fig. 3. Example of Standard Time Shift

Now we show a simple example of a proof with standard time shift [1]. Suppose we want to prove that the lower bound for the write operation is $x$. Assume in contradiction, there exists a correct implementation of a read-write register in which the worst-case time complexity of the write operation is less than $x$. First we let two processes $p_{i}$ and $p_{j}$ execute operations as Fig. 3(a). In this run, write(1) is invoked immediately after write $(0)$ completes. For this run, operation sequence write $(0) \circ$ write $(1) \circ$ read(1) is legal and respects the real time sequence. Then we use the standard time shift to shift the local clock in process $p_{i}$ by $2 x$ and all the message delays are still admissible after the standard shift, as shown in Fig. 3(b). Because the executions of operations are exactly the same as before the standard time shift, the read operation still returns 1 . But after the standard time shift, there is no operation sequence of the three operations which is legal and respects the real time sequence, because write( 0$)$ is invoked after write(1) completes. Therefore, the assumed correct implementation does not exist. 
In the example above, to prove a lower bound $x$, we shift the local clock of $p_{i}$ by $2 x$. On one side, we can imagine that by shifting with larger amounts, we can prove a larger lower bound. On the other side, the new message delays must fall into the range $[d-u, d]$ and they are determined by the original message delays and shift amounts, meaning that the shift amounts are limited, otherwise the new message delays are invalid. For instance, in the previous example, by setting $x>u / 2$, the new message delays are increased or decreased from old message delays by more than $u$. Since the old message delays are within $[d-u, d]$, the new message delays must be invalid. To overcome the limitation of shift amounts, we develop the modified time shift in the following section.

\section{B. Modified Time Shift}

We develop the modified time shift from the standard one. The intuition is to prove larger lower bounds by shifting the local clocks with larger amounts and the general idea is as below.

In the standard time shift, all the messages delays are still valid in the shifted run. In the modified shift of a run, depending on the original message delays and the time shift amounts, the new message delays may be invalid. The weaker guarantee allows us to shift by larger amounts and thus prove larger lower bounds than before. But we must get rid of the invalid messages. We chop the shifted run to get a prefix of it, such that the invalid messages are not received in the prefix and so the prefix is admissible. After the the run is chopped, there is no invalid message, but the run is not a complete run. So we need to extend the chopped run to a complete run.

With the three steps (1) modified time shift, (2) chopping, and (3) extending, we get a new run, whose prefix is the same as the original run. Therefore if an operation 
is invoked and responds within the prefix, the operation returns the same value as in the original run. In this way, we can make use of the modified time shift in a similar way as the standard time shift.

Now we illustrate the differences between standard time shift and modified time shift, and the three steps by simple examples in Fig. 4.

Part (a) in Fig. 4 is an example of the standard time shift. Before the time shift, $d_{i, j}=d_{j, i}=d-u / 2$. clock_shift $(i)=0$ and $\operatorname{clock} \_s h i f t(j)=u / 2$. According to formula (1), after the standard time shift, $d_{i, j}^{\prime}=d-u / 2-0+u / 2=d$, and $d_{j, i}^{\prime}=d-u / 2-u / 2+0=d-u$, both of which are within the range $[d-u, d]$ and admissible.

Part (b) in Fig. 4 is an example of the modified time shift. Different from part (a), $d_{i, j}=d_{j, i}=d$.

- Step 1: We shift the local clocks in processes $p_{i}$ and $p_{j}$ with $\operatorname{clock} \_$shift $(i)=0$ and clock $_{-} \operatorname{shift}(j)=u$. According to formula $(1), d_{i, j}^{\prime}$ is $d+u$, which is not admissible and $d_{j, i}^{\prime}$ is $d-u$.

- Step 2: To get an admissible prefix of the shifted run, we consider the view in each process: there are two messages between $p_{i}$ and $p_{j}$. The first message is sent from $p_{j}$ to $p_{i}$ and does not violate message delay constraints. The second message is sent from $p_{i}$ to $p_{j}$ and is not admissible. For process $p_{j}$, we chop the view so that the prefix ends before the clock time $T_{4}^{\prime}$, because the invalid message is not received before $T_{4}^{\prime}$. In process $p_{i}$, since the view of $p_{j}$ is chopped to end before $T_{4}^{\prime}$ and the message sent at $T_{4}^{\prime}$ costs at least $d-u$ to be received by $p_{i}$ (clock time $T_{6}^{\prime}$ ), the view of $p_{i}$ is chopped to end before $T_{6}^{\prime}$; otherwise the message which is sent at $T_{4}^{\prime}$ from $p_{j}$ to $p_{i}$ is sent after the prefix but received in the prefix. 


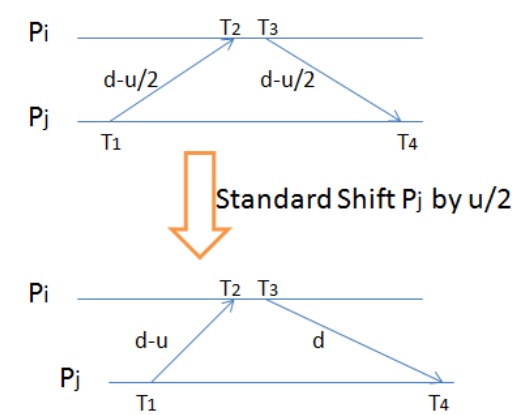

(a) Standard Time Shift

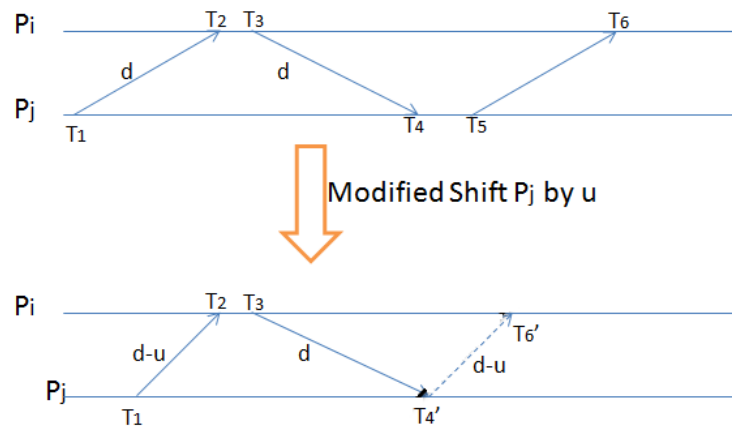

(b) Modified Time Shift

Fig. 4. Standard Time Shift and Modified Time Shift

- Step 3: Extend the prefix to a complete run by letting the second message be received at $T_{4}^{\prime}$ (message delivery costs $d$, which is admissible). Then all the messages are received and the run is complete.

\section{Chopping and Extending}

Let $R$ be a run with pairwise uniform message delays $\left\{d_{k 1, k 2}: p_{k 1} \in \Pi, p_{k 2} \in \Pi\right\}$ such that the maximum clock skew is bounded by $\epsilon$ and exactly one message delay $d_{i, j}$ is invalid. Let $m$ be the first message sent from $p_{i}$ to $p_{j}$ in $R$, say at real time $t_{s}$. Let $t^{*}=t_{s}+\min \left\{d_{i, j}, \delta\right\}(\delta$ is a parameter in the range $[d-u, d])$. Let $V_{j}$ be the prefix of $p_{j}$ 's timed view in $R$ that ends just before real time $t^{*}$ (the cross sign in Fig. 5). For each $k \neq j$, let $V_{k}$ be the prefix of $p_{k}$ 's timed view in $R$ that ends just before real time $t^{*}+D_{j, k}$, where $D_{j, k}$ is the shortest path distance from $p_{j}$ to $p_{k}$ in the complete directed graph whose node set is $\Pi$ and in which the edge from $p_{k 1}$ to $p_{k 2}$ is weighted with $d_{k 1, k 2}$ for all $p_{k 1}, p_{k 2} \in \Pi$. Then $\operatorname{chop}(R, \delta)$ is defined to be $\left\{V_{i}: p_{i} \in \Pi\right\}$.

Lemma B.1 If $R$ is a run with pairwise uniform message delays that satisfies admissibility except that exactly one message delay is invalid, then $R^{\prime}=\operatorname{chop}(R, \delta)$ is 
an admissible run for all $\delta \in[d-u, d]$.

Proof. First, $R^{\prime}$ is a run, because

(1) every element in $R^{\prime}$ is chopped from a timed view in $R$, which means it is also a timed view; and

(2)all the messages received in $R^{\prime}$ are sent in $R^{\prime}$. Suppose in contradiction, there is a message $m^{\prime}$ sent from $p_{k_{1}}$ to $p_{k_{2}}$, which is sent after $t^{*}+D_{j, k_{1}}$ and received before $t^{*}+D_{j, k_{2}}$. Then $D_{j, k_{2}}-D_{j, k_{1}}>d_{k_{1}, k_{2}}$. So $D_{j, k_{1}}+d_{k_{1}, k_{2}}<D_{j, k_{2}}$ and $D_{j, k_{2}}$ is not the shortest path distance from $p_{j}$ to $p_{k_{2}}$.

Second, $R^{\prime}$ is admissible, because:

1. All the messages received in $R^{\prime}$ have delays in the range $[d-u, d]$. This is because there is only one invalid message delay $d_{i, j}$ and we cut the view $V_{j}$ to end before the invalid message is received.

2. For each message which is sent at real time $t$ in $R^{\prime}$ but not received in $R^{\prime}$, the view of the recipient is chopped before $t+d$. For the message $m$ from $p_{i}$ to $p_{j}$, because $t^{*} \leq t_{s}+\delta \leq t_{s}+d, p_{j}$ ends before $t_{s}+d$. For all other messages, because their message delays are no larger than $d$, if they are not received in $R^{\prime}$, the recipient ends before the sending time plus $d$.

3. $R^{\prime}$ has the same clock functions as $R$, for which the maximum clock skew is no larger than $\epsilon$.

Remark: $t^{*}=t_{s}+\min \left\{d_{i, j}, \delta\right\}(d-u \leq \delta \leq d)$ ensures $t^{*} \leq t_{s}+\delta$, which means $V_{j}$ ends before $t_{s}+\delta$ in $R^{\prime}$. When $R^{\prime}$ is extended, we can let the message delay from $p_{i}$ to $p_{j}$ fall into the range $[\delta, d]$. Since $m$ is sent at real time $t_{s}, m$ is received after 
$t_{s}+\delta$ and so after $V_{j}$ ends. Then $m$ still does not affect $R^{\prime}$. And since the message delay is admissible within $[\delta, d]$, we get an admissible extension of $R^{\prime}$.

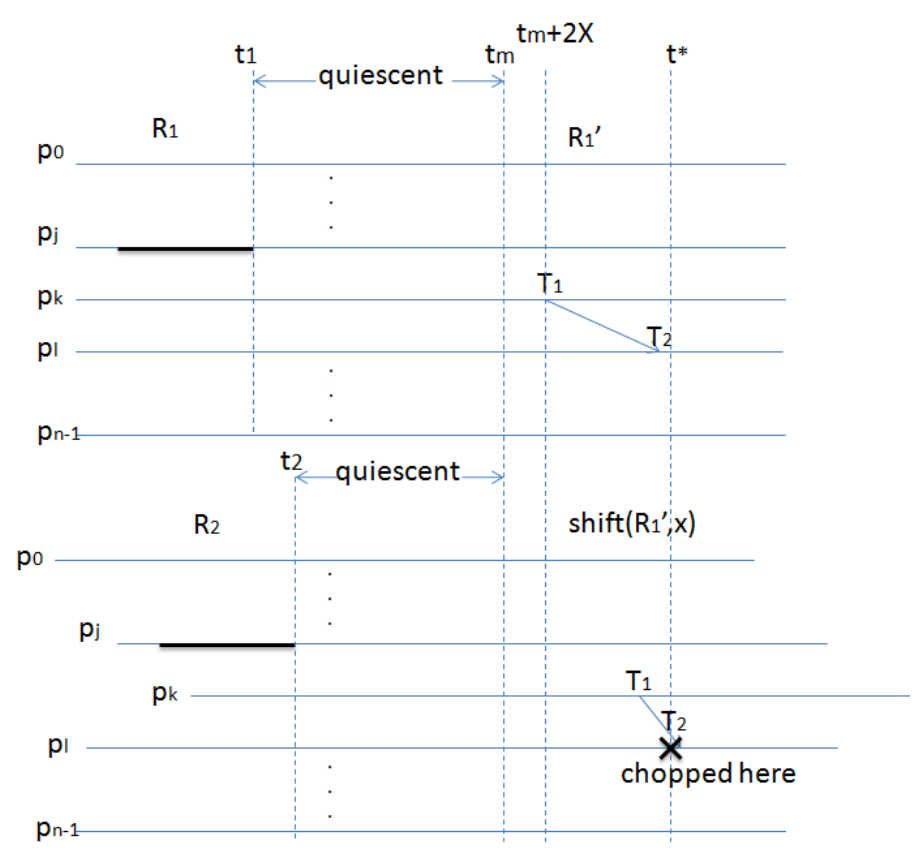

Fig. 5. Chopping and Appending to an Initialized Run

\section{Appending to an Initialized Run}

If the chopped run is not initialized, there might be a problem about whether it is appendable to an initialized run. To answer this question, we have two claims below.

Given a legal sequence $\rho$ of finite operations on a shared object, define $\mathscr{R}_{\rho}$ to be the set of all runs $R$ satisfying the following conditions:

- $R$ is an initialized, complete and admissible run;

- process $p_{0}$ executes operations in $\rho$ sequentially, and no other process executes any operation; 
- the first operation is invoked at real time $t_{0}$; and

- each operation (except the first one) is invoked immediately after the previous operation responds.

The bounded time operation and bounded quiescence properties (from Chapter III) show the following claim:

Claim B.2 For every $R \in \mathscr{R}, R$ is finite and every process is quiescent by real time $t_{q}=t_{0}+|\rho| \times B_{o p}+B_{q}$.

Let $R_{1}$ be any run in $\mathscr{R}$. Let $R_{1}^{\prime}$ be an admissible run which is appendable to $R_{1}$ and starts at real time $t_{q}+2 X$, where $X$ is a real number which bounds the shift amounts in the following claim (Fig. 5).

Claim B.3 For every n-vector $\vec{x}$ with $\left|x_{i}\right| \leq X, 0 \leq i \leq n-1$, there exists a run $R_{2} \in \mathscr{R}$ such that shift $\left(R_{1}^{\prime}, \vec{x}\right)$ is appendable to $R_{2}$.

Proof. Let $R_{2}$ have clock functions shifted from those of $R_{1}$ by $\vec{x}$. In $R_{2}$, let all processes start with their initial states and let $p_{0}$ invoke the operations in $\rho$ starting at real time $t_{0}$ without any time gap between two operations. Then $R_{2}$ is one element of $\mathscr{R}$. $\operatorname{shift}\left(R_{1}^{\prime}, \vec{x}\right)$ is appendable to $R_{2}$, because:

1. According to the definition of appendable runs, for each process $p_{i}$, the clock functions in $R_{1}^{\prime}$ are the same as the clock functions in $R_{1}$. Because clock times in $R_{2}$ are shifted by $\vec{x}$ from $R_{1}$, shift $\left(R_{1}^{\prime}, \vec{x}\right)$ has the same clock functions as in $R_{2}$.

2. Because $R_{1}^{\prime}$ starts after $t_{q}+2 X$, $\operatorname{shift}\left(R_{1}^{\prime}, \vec{x}\right)$ starts after $t_{q}+X$. So for each process, the first clock time in $\operatorname{shift}\left(R_{1}^{\prime}, \vec{x}\right)$ is later than the last clock time in $R_{2}$. 
3. Based on the assumption of History-Obliviousness, every process $p_{i}$ ends with the same state in $R_{1}$ and $R_{2}$, which is the same as the first state in $R_{1}^{\prime}$ and in $\operatorname{shift}\left(R_{1}^{\prime}, \vec{x}\right)$.

Corollary B.4 If there is only one invalid message delay in shift $\left(R_{1}^{\prime}, \vec{x}\right),\left|x_{i}\right| \leq X$, $R_{1}^{\prime}$ is appendable to the run $R_{1} \in \mathscr{R}_{\rho}$ and starts after $t_{q}+X$, then there exists a run $R_{2} \in \mathscr{R}_{\rho}$, such that chop $\left(\operatorname{shift}\left(R_{1}^{\prime}, \vec{x}\right), \delta\right)$ is appendable to $R_{2}$.

Note: The assumption of "Bounded Operation", "Bounded Quiescence" and "History Obliviousness" are used in Claim B.3. This claim is used in the following theorems to exclude the impact of previous operation history. The operation response should depend on the initialization of the object, sequential operation history and other concurrent operations. The details of previous operations such as execution times should be unrelated. Here we consider the objects with initialization constraints that the object can only be initialized with certain specific values. If there is no constraint in the initialization, we can initialize the object with the state after the previous operation sequence. In this way, the impact of the previous operation execution details has already been excluded when the object is initialized.

C. Strongly Immediately Non-self-commuting Operation Types

Theorem C.1 For every strongly immediately non-self-commuting operation type $O P,|O P| \geq d+m$ time $(m=\min \{\epsilon, u, d / 3\})$ for any linearizable shared object implemented in an $n$-process $(n \geq 3)$ message passing system with message delay bound $[d-u, d]$ and $\epsilon$-bounded clocks.

Proof. Suppose, in contradiction that on a linearizable shared object, there exists 
an operation type $O P$ which is strongly immediately non-self-commuting, but the operations of this type cost less than $d+m$ time. From the definition of strongly immediately non-self-commuting, there exist an operation sequence $\rho$ on this object, operations $o p_{1} \in O P$ and $o p_{2} \in O P$, such that

(1) $\rho \circ o p_{1}$ is legal

(2) $\rho \circ o p_{2}$ is legal

(3) $\rho \circ o p_{1} \circ o p_{2}$ is illegal

(4) $\rho \circ o p_{2} \circ o p_{1}$ is illegal

We assume that $o p_{1}=O P\left(\arg _{1}, \operatorname{ret}_{1}\right)$ and $o p_{2}=O P\left(\arg _{2}, \operatorname{ret}_{2}\right)$.

The counter-example is constructed with the following 4 steps (Proof structure refers to Figure 6). In the statements of runs in each step, we ignore the operation sequence $\rho$, assuming the operations in each run are invoked after all operations in $\rho$ have responded and every process is quiescent for a long time.

Step 1: Let $p_{i}$ and $p_{j}$ be two specific processes and $p_{k}$ denotes any other process. In $R_{1}$ :

- All the processes except process $p_{j}$ have the same local clock function; $p_{j}$ 's local clock function is $m$ later than the other processes;

- The message delays are pairwise uniform with $d_{i, k}=d_{i, j}=d_{j, i}=d_{k, j}=d$; and with $d_{k, i}=d_{j, k}=d-m$. The message delay from $p_{k}$ to $p_{k^{\prime}}$ for any $k \neq i, j$ and $k^{\prime} \neq i, j$ is any valid delay $\Delta t$ where $d-u \leq \Delta t \leq d$ (Fig. 7 (a)).

In run $R_{1}, p_{i}$ executes the operation $o p_{1}^{\prime} \in O P$ with argument $\arg _{1}$ invoked at real time $t$ (local time $T$ ). Then process $p_{j}$ executes operation $o p_{2}^{\prime} \in O P$ with argument $\arg _{2}$ invoked at real time $t+m$ (local time $T$ ). No other operations are invoked.

In run $R_{1}^{\prime}$, every process has the same local clock as in $R_{1}$ and the message delays 


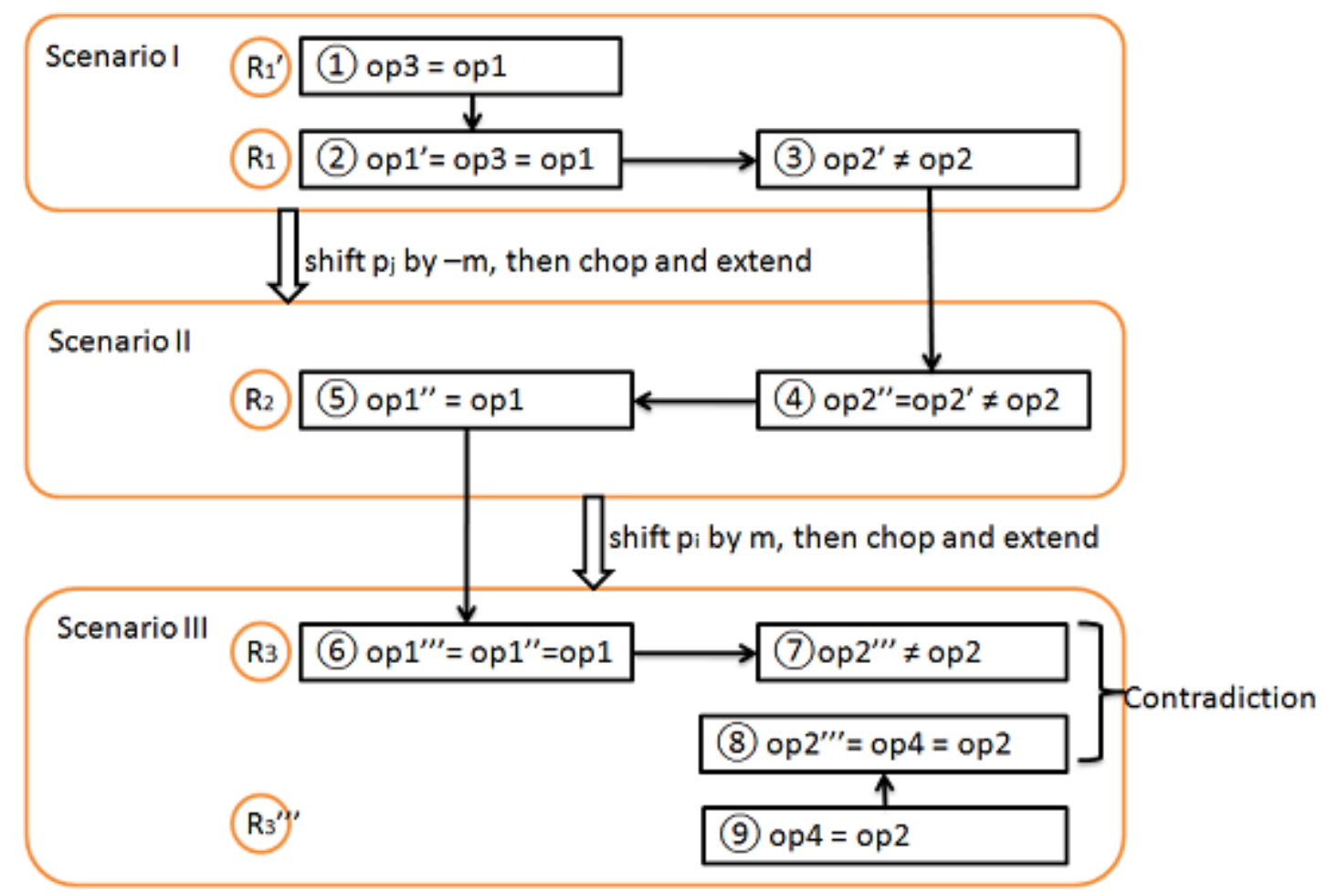

Fig. 6. Proof Structure of Theorem C.1

are also the same as in $R_{1} ; p_{i}$ executes an operation $o p_{3} \in O P$ with argument $\arg g_{1}$ invoked at real time $t$ (local time $T$ ). No other operations are invoked.

Then runs $R_{1}$ and $R_{1}^{\prime}$ are both admissible because:

- The maximum local clock skew is $m=\min \{\epsilon, u, d / 3\}$, which is not larger than $\epsilon$.

- Because $m=\min \{\epsilon, u, d / 3\}$ is not larger than $u$, letting message delay be $d-m$ or $d$ is admissible.

Step 1.1: We will show $o p_{1}^{\prime}=o p_{1}$ (1) and (2) in Fig. 6), i.e., that the value returned in $R_{1}$ for $o p_{1}^{\prime}$ is ret $_{1}$.

In run $R_{1}^{\prime}$, by the definition of a deterministic object, op $p_{3}$ has to return ret r $_{1}$ 


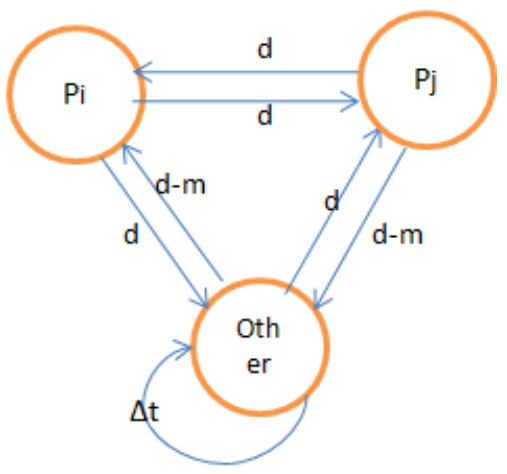

(a) Message Delays in $R_{1}$ and $R_{1}^{\prime}$

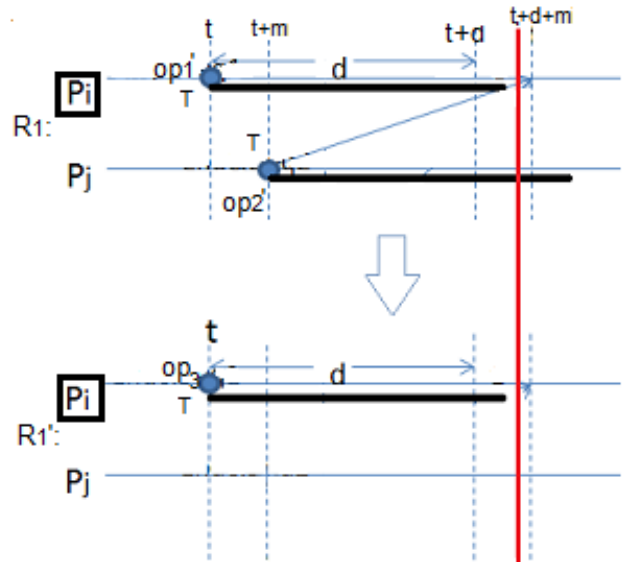

(b) $R_{1}$ and $R_{1}^{\prime}$

Fig. 7. Step 1 in Theorem C.1

because there is no other concurrent operations. Therefore $o p_{3}=o p_{1}$.

In run $R_{1}$, there are two concurrent operations $o p_{1}^{\prime}$ and $o p_{2}^{\prime}$. Since $m \leq d / 3$, it follows that $D_{j, i} \geq d$, meaning that $p_{i}$ cannot "learn about" $o p_{2}^{\prime}$ until real time $t+d+m$. Then before $t+d+m$, the view of $p_{i}$ is the same in $R_{1}$ and in $R_{1}^{\prime}$. Therefore, $o p_{1}^{\prime}$ returns the same value ret $_{1}$ as $o p_{3}$ in $R_{1}^{\prime}$, and so $o p_{1}^{\prime}=o p_{3}=o p_{1}$. (Fig. 7(b))

Step 1.2: We will show $o p_{2}^{\prime} \neq o p_{2}(3)$ in Fig. 6).

By the assumed correctness of the algorithm, at least one of the below must be legal: $\rho \circ o p_{1}^{\prime} \circ o p_{2}^{\prime}$ and $\rho \circ o p_{2}^{\prime} \circ o p_{1}^{\prime}$. According to the definition of strongly immediately non-self-commuting operation, $\rho \circ o p_{1} \circ o p_{2}$ and $\rho \circ o p_{2} \circ o p_{1}$ are both illegal. We already proved $o p_{1}^{\prime}=o p_{1}$. If $o p_{2}^{\prime}=o p_{2}$, it would mean neither $\rho \circ o p_{1}^{\prime} \circ o p_{2}^{\prime}$ nor $\rho \circ o p_{2}^{\prime} \circ o p_{1}^{\prime}$ is legal, contradicting that at least one of $\rho \circ o p_{1}^{\prime} \circ o p_{2}^{\prime}$ and $\rho \circ o p_{2}^{\prime} \circ o p_{1}^{\prime}$ must be legal.

Step 1.3: We will show that $\rho \circ o p_{2}^{\prime} \circ o p_{1}^{\prime}$ is illegal and thus $\rho \circ o p_{1}^{\prime} \circ o p_{2}^{\prime}$ must be legal. 
Because $o p_{2}^{\prime} \neq o p_{2}$ and $\rho \circ o p_{2}$ is legal, based on the definition of deterministic object, $\rho \circ o p_{2}^{\prime}$ must be illegal, which means $\rho \circ o p_{2}^{\prime} \circ o p_{1}^{\prime}$ is illegal.

Step 2: Define run $R_{2}^{\prime}=\operatorname{shift}\left(R_{1}, \vec{x}\right)$, where $x_{j}=-m$ and $x_{k}=0$ for all $k \neq j$, which means $p_{i}$ executes $o p_{1}^{\prime}$ with argument $\arg _{1}$ invoked at real time $t$ (local time $T$ ), and $p_{j}$ executes $o p_{2}^{\prime}$ with argument $\arg _{2}$ invoked at real time $t$ (local time $T$ ).

Now we apply Lemma B.1 here. According to formula (1), $d_{j, i}^{\prime}$ in $R_{2}^{\prime}$ is $d+m$, and it is the only invalid message delay. Because $o p_{1}^{\prime}$ and $o p_{2}^{\prime}$ are both invoked at real time $t$ in $R_{2}^{\prime}$, the earliest possible message from $p_{j}$ to $p_{i}$ is sent no earlier than $t$. By setting $\delta$ to $d-m$, we get $t^{*}=t+\min \{d+m, d-m\}=t+d-m$. Let $R_{2}^{\prime \prime}=\operatorname{chop}\left(R_{2}^{\prime}, d-m\right)$. By Lemma B.1, $R_{2}^{\prime \prime}$ is admissible. (In Fig. 8, the cross signs are where each view is chopped.)

We can extend $R_{2}^{\prime \prime}$ to a complete admissible run $R_{2}$ by letting messages from $p_{j}$ to $p_{k} \operatorname{cost} \delta=d-m$ time. The invocations of $o p_{1}^{\prime}$ and $o p_{2}^{\prime}$ happen in $R_{2}^{\prime \prime}$, but the responses of them may not. So in $R_{2}, p_{i}$ invokes an operation $o p_{1}^{\prime \prime} \in O P$ with $\arg _{1}$ at real time $t$, and $p_{j}$ invokes an operation $o p_{2}^{\prime \prime} \in O P$ with $\arg _{2}$ at real time $t$, but the response of $o p_{1}^{\prime \prime}$ (respectively $o p_{2}^{\prime \prime}$ ) may be different from that of $o p_{1}^{\prime}$ (respectively $\left.o p_{2}^{\prime}\right)$.

However, we will now prove that $o p_{1}^{\prime \prime}=o p_{1}^{\prime}=o p_{1}$ and $o p_{2}^{\prime \prime}=o p_{2}^{\prime}$.

Step 2.1: First we show $o p_{2}^{\prime \prime}=o p_{2}^{\prime}$ (4) in Fig. 6).

For process $p_{j}, D_{i, j}^{\prime}=d_{i, j}=d-m$, because $d-m$ is the smallest message delay in $R_{2}^{\prime \prime}$. Then $t^{*}+D_{i, j}^{\prime}=t+d-m+d-m \geq t+d+m$, and $V_{j} \in R_{2}^{\prime \prime}$ ends after $t+d+m$. Because $o p_{2}^{\prime \prime}$ returns before $t+d+m, o p_{2}^{\prime \prime}$ responds within $R_{2}^{\prime \prime}$, which is a prefix of $\operatorname{shift}\left(R_{1}, \vec{x}\right)$. Therefore, $o p_{2}^{\prime \prime}$ returns the same value as $o p_{2}^{\prime}$ and $o p_{2}^{\prime \prime}=o p_{2}^{\prime}$.

Step 2.2: Now we show $o p_{1}^{\prime \prime}=o p_{1}$ (5) in Fig. 6).

Since $o p_{2}^{\prime \prime} \neq o p_{2}$ and $\rho \circ o p_{2}$ is legal, by the definition of a deterministic object, $\rho \circ o p_{2}^{\prime \prime}$ is illegal. So $\rho \circ o p_{2}^{\prime \prime} \circ o p_{1}^{\prime \prime}$ is illegal, and then by the assumed correctness of the 


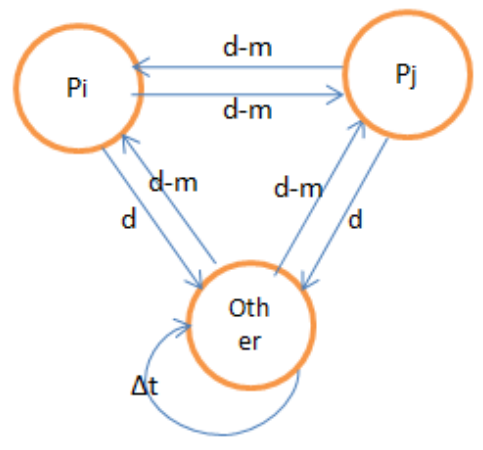

(a) Message Delays in $R_{2}$

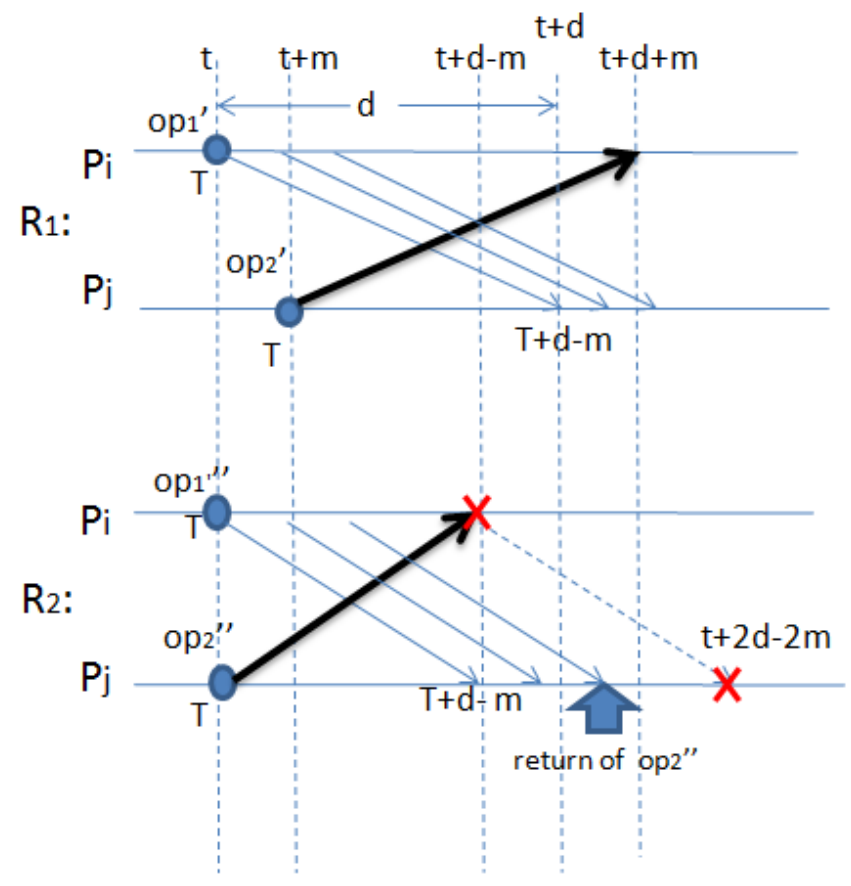

(b) $R_{1}$ and $R_{2}$

Fig. 8. Step 2 in Theorem C.1

algorithm, $\rho \circ o p_{1}^{\prime \prime} \circ o p_{2}^{\prime \prime}$ must be legal. Considering $\rho \circ o p_{1}$ is legal and $o p_{1}^{\prime \prime}$ has the same argument as $o p_{1}, o p_{1}^{\prime \prime}=o p_{1}$, otherwise it contradicts the definition of deterministic object.

Step 3: Let run $R_{3}^{\prime}=\operatorname{shift}\left(R_{2}, \vec{x}\right)$, where $x_{i}=m$ and $x_{k}=0$ for all $k \neq i$, which means $p_{i}$ executes $o p_{1}^{\prime \prime}$ with argument $\arg _{1}$ invoked at real time $t+m$ (local time $T$ ), and $p_{j}$ executes $o p_{2}^{\prime \prime}$ with argument $\arg _{2}$ invoked at real time $t$ (local time $\mathrm{T}$ ).

Now we apply Lemma B.1 here. According to formula (1), $d_{i, j}^{\prime \prime}$ is $d-2 m$, which may violate the message delay constraint. The earliest possible message from $p_{i}$ to $p_{j}$ is sent no earlier than real time $t+m$, because $o p_{1}^{\prime \prime}$ is invoked at real time $t+m$ in $R_{3}^{\prime}$, and $o p_{2}^{\prime \prime}$ is invoked at real time $t$, meaning the earliest message to $p_{i}$ is received no earlier than $t+d-m$, which is larger than $t+m$ because $m \leq d / 3$. Then still 
by setting $\delta=d-m$, we get $t^{*}=t+m+\min \{d-2 m, d-m\}=t+d-m$. Let $R_{3}^{\prime \prime}=\operatorname{chop}\left(R_{3}^{\prime}, d-m\right)$. By Lemma B.1, $R_{3}^{\prime \prime}$ is admissible. (In Fig. 9, the cross signs are where each view is chopped.)

We can extend $R_{3}^{\prime \prime}$ to a complete admissible run $R_{3}$ by letting messages sent from $p_{i}$ to $p_{j} \operatorname{cost} d>\delta$ time. Similarly to Step 2, the invocations of $o p_{1}^{\prime \prime}$ and $o p_{2}^{\prime \prime}$ happen in $R_{2}^{\prime \prime}$, but the responses of them may not. So $p_{i}$ invokes an operation $o p_{1}^{\prime \prime \prime} \in O P$ with $a r g_{1}$ at real time $t+m$, and $p_{j}$ invokes an operation $o p_{2}^{\prime \prime \prime} \in O P$ with $a r g_{2}$ at real time $t$, but the response of $o p_{1}^{\prime \prime \prime}$ (respectively $o p_{2}^{\prime \prime \prime}$ ) may be different from that of $o p_{1}^{\prime \prime}$ (respectively $\left.o p_{2}^{\prime \prime}\right)$.

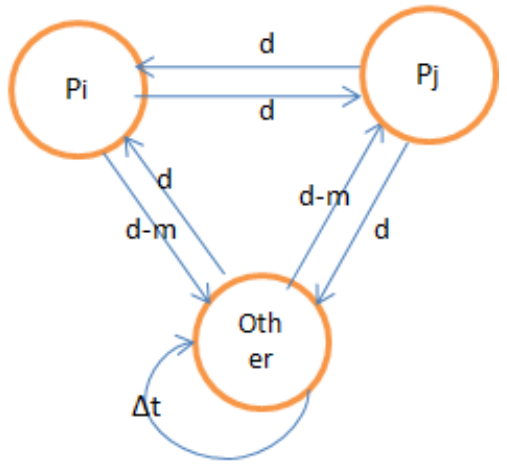

(a) Message Delays in $R_{3}$ and $R_{3}^{\prime}$

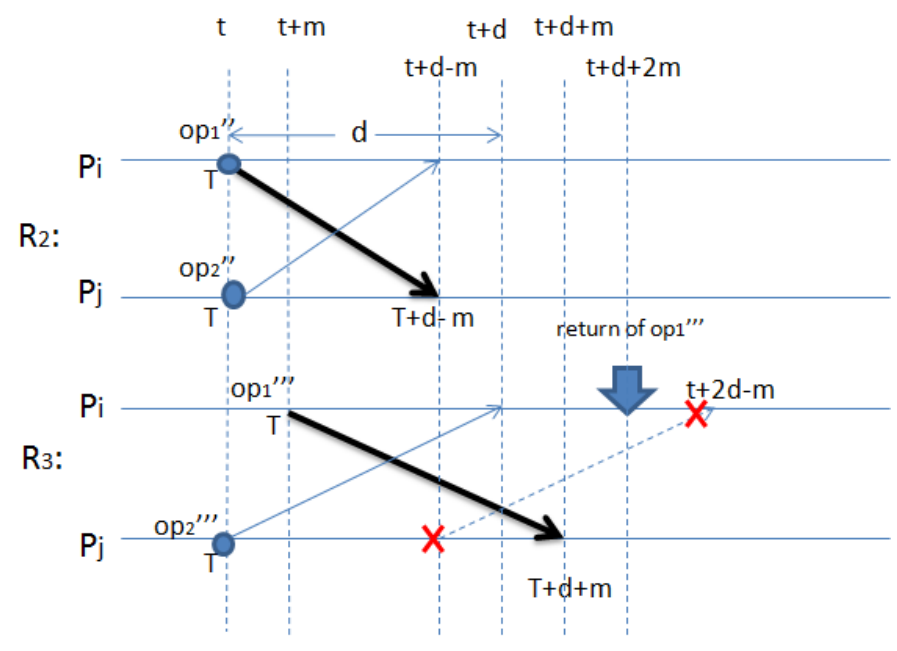

(b) $R_{2}$ and $R_{3}$

Fig. 9. Step 3 in Theorem C.1

Similarly to step 2.2, we can prove that $o p_{1}^{\prime \prime \prime}=o p_{1}^{\prime \prime}=o p_{1}($ (6) in Fig. 6). Then similarly to the last two paragraphs in Step 1, we can show that $o p_{2}^{\prime \prime \prime} \neq o p_{2}((7)$ in Fig. 6) and $\rho \circ o p_{1}^{\prime \prime \prime} \circ o p_{2}^{\prime \prime \prime}$ is legal.

Step 4: We define a run $R_{3}^{\prime \prime \prime}$ as follows. Let all processes in run $R_{3}^{\prime \prime \prime}$ have the 
same clock functions and message delays as in $R_{3}$. Then $R_{3}^{\prime \prime \prime}$ is also admissible.

In run $R_{3}^{\prime \prime \prime}: p_{j}$ executes $o p_{4}$ with argument $a r g_{2}$ invoked at real time $t$. No other operations are invoked.

In $R_{3}^{\prime \prime \prime}$, because there is only one operation $o p_{4}$, by the definition of a deterministic object, $o p_{4}$ must return $r e t_{2}$. So $o p_{4}=o p_{2}\left(9\right.$ in Fig. 6). Next we prove $o p_{2}^{\prime \prime \prime}=o p_{4}=$ $o p_{2}(8)$ in Fig. 6).

Similar to Step 1, because it is impossible for $p_{j}$ to get any information about $o p_{1}^{\prime \prime \prime}$ before $o p_{2}^{\prime \prime \prime}$ returns, $p_{j}$ has the same local view in $R_{3}$ and $R_{3}^{\prime \prime \prime}$ until $o p_{2}^{\prime \prime \prime}$ returns. Therefore, $o p_{2}^{\prime \prime \prime}$ in $R_{3}$ returns what $o p_{4}$ returns and $o p_{2}^{\prime \prime \prime}=o p_{4}=o p_{2}$. This contradicts the conclusion in Step 3 that $o p_{2}^{\prime \prime \prime} \neq o p_{2}$.

\section{Eventually Non-self-last-permuting Operation Types}

By studying the commutativity property, the time lower bounds for eventually nonself-commuting operation types such as write, push, enqueue have been proved to be $u / 2$ [3]. Considering the scale of the ever growing shared object system, we extend commutativity to permutations for more than two concurrent operations. In the following theorem, we prove using the standard shift technique, that for a group of characterized operation types, which also includes write, push and enqueue, the lower bound of operations in this group is $(1-1 / k) u(k \leq n$ is a positive integer determined by the property of the operations). More specifically, for those immediately non-selflast-permuting operations such as write on a register, push on a stack and enqueue on a queue, $k=n$ and the lower bounds for them are $(1-1 / n) u$.

Theorem D.1 Let $O P$ be an immediately self-commuting operation type, for which there exist an integer $k \geq 2$, an operation sequence $\rho$, and $k$ distinct operations $o p_{i}\left(\arg _{i}\right.$, ret $\left._{i}\right) \in O P, 0 \leq i \leq k-1$, such that for all $0 \leq i \leq k-1, \rho \circ o p_{i}$ is 
legal, and for any two legal permutations $\pi$ and $\pi^{\prime}$ of $\left\{\right.$ op $\left._{i}: 0 \leq i \leq k-1\right\}$ with last $(\pi) \neq \operatorname{last}\left(\pi^{\prime}\right), \pi$ and $\pi^{\prime}$ are not equivalent. Then the time complexity of OP in any implementation of $O P$ in a system with $n \geq k$ processes, $\epsilon$-bounded clocks, and message delays in $[d-u, d]$ is at least $(1-1 / k) u$.

Proof. Suppose in contradiction there is an implementation of such an operation type $O P$ in such a system with time complexity less than $(1-1 / k) u$. Since $\rho \circ o p_{i}$ is legal for each $i$, and $O P$ is immediately self-commuting, a simple inductive proof shows that for any permutation $\pi$ of the $k$ operations, $\rho \circ \pi$ is legal.

Step 1: We construct run $R_{1}$ with the process set $\Pi$ in which:

- all the processes have the same clock function;

- For all $0 \leq i, j \leq k-1, i \neq j, d_{i, j}=d-[(i-j) \% k / k] \cdot u$. For all $k \leq l \leq n-1$, all $0 \leq l^{\prime} \leq n-1$ and $l \neq l^{\prime}, d_{l, l^{\prime}}=d_{l^{\prime}, l}=d-u / 2$ (shown in Fig. 10).
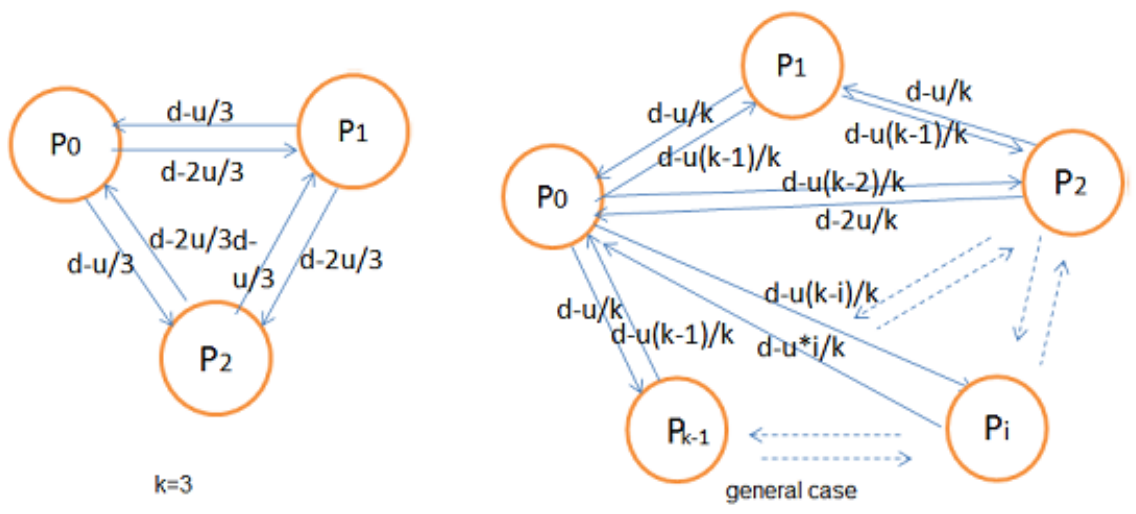

Fig. 10. Message Delays in $R_{1}\left(R_{1}^{\prime}\right)$

In run $R_{1}$ (Fig. 11), each process $p_{i}(0 \leq i \leq k-1)$ executes $o p_{i}$ at real time $t$ (local clock $T$ ). No other operations are invoked. Then each $p_{i}$ ends before $t+u$. 
$R_{1}$ is admissible because:

1. The maximum local clock skew is 0 , smaller than $\epsilon$.

2. Because $d-u<d-j \cdot u / k \leq d$, all the message delays are admissible.

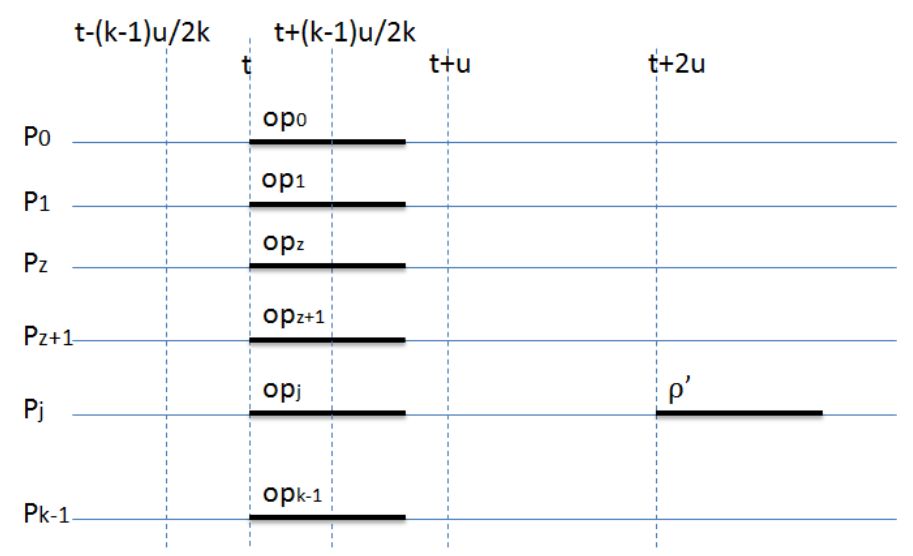

Fig. 11. Run $R_{1}\left(R_{1}^{\prime}\right)$

Step 1.1: By the definition of the deterministic object and the assumed correctness of the algorithm, there exists at least one legal permutation $\pi$ for the $k$ operations in $R_{1}$, such that for any operation sequence $\rho^{*}$, if $\rho \circ \pi \circ \rho^{*}$ is legal, then when the operations in $\rho^{*}$ are invoked sequentially after $t+2 u$ (after all the operations in $R_{1}$ return), each operation returns the same value as in $\rho^{*}$.

Step 1.2: Without loss of generality, assume $\operatorname{last}(\pi)=o p_{z}$.

Step 2: Let run $R_{2}=\operatorname{shift}\left(R_{1}, \vec{x}\right)$ where $x_{i}=[-(k-1) / 2+(z-i) \% k / k] \cdot u$ $(0 \leq i \leq k-1)$. According to formula $(1), d_{i, j}^{\prime}=d-[(i-j) \% k / k] \cdot u-[-(k-1) / 2+$ $(z-i) \% k / k] \cdot u+[-(k-1) / 2+(z-j) \% k / k] \cdot u=d-[(i-j) \% k / k] \cdot u-[(z-i) \% k / k] \cdot$ $u+[(z-j) \% k / k] \cdot u$ (shown in Fig. 13 and Fig. 14).

- If $i<j \leq z, d_{i, j}^{\prime}=d-[(i-j+k) / k] \cdot u-[(z-i) / k] \cdot u+[(z-j) / k] \cdot u=d-u$. 
- If $i \leq z<j, d_{i, j}^{\prime}=d-[(i-j+k) / k] \cdot u-[(z-i) / k] \cdot u+[(z-j+k) / k] \cdot u=d$.

- If $z<i<j, d_{i, j}^{\prime}=d-[(i-j+k) / k] \cdot u-[(z-i+k) / k] \cdot u+[(z-j+k) / k] \cdot u=d-u$.

- If $j<i \leq z, d_{i, j}^{\prime}=d-[(i-j) / k] \cdot u-[(z-i) / k] \cdot u+[(z-j) / k] \cdot u=d$.

- If $j \leq z<i, d_{i, j}^{\prime}=d-[(i-j) / k] \cdot u-[(z-i+k) / k] \cdot u+[(z-j) / k] \cdot u=d-u$.

- If $z<j<i, d_{i, j}^{\prime}=d-[(i-j) / k] \cdot u-[(z-i+k) / k) \cdot u+[(z-j+k) / k] \cdot u=d$.

(A simple example of this shift is in Fig. 12.)

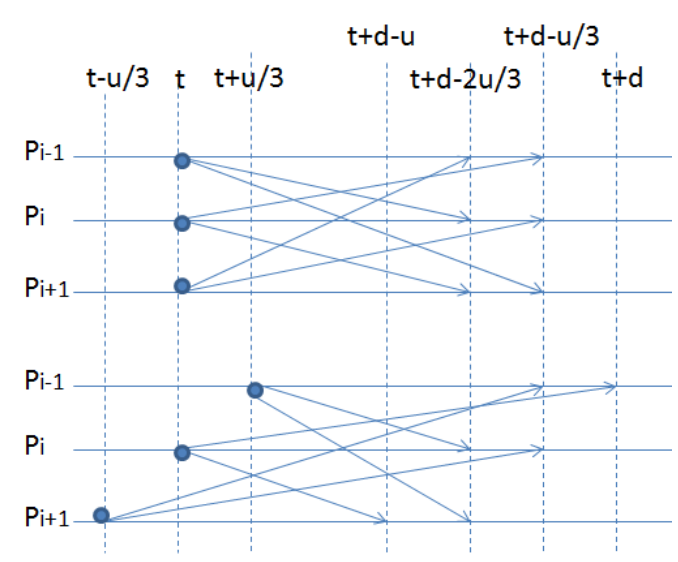

Fig. 12. Example of Standard Time Shift of $R_{1}$

$R_{2}$ is admissible because:

1. The maximum local clock skew is $[-(k-1) / 2+(k-1)] / k \cdot u-[-(k-1) / 2 k] u=$ $(1-1 / k) u$, no larger than $\epsilon$, because the optimal $\epsilon$ is $(1-1 / n) u \geq[1-(k-1) / k] u$.

2. All the message delays are admissible:

- For $0 \leq i, j \leq k-1, d_{i, j}^{\prime}$ is either $d$ or $d-u$, which are both admissible. 

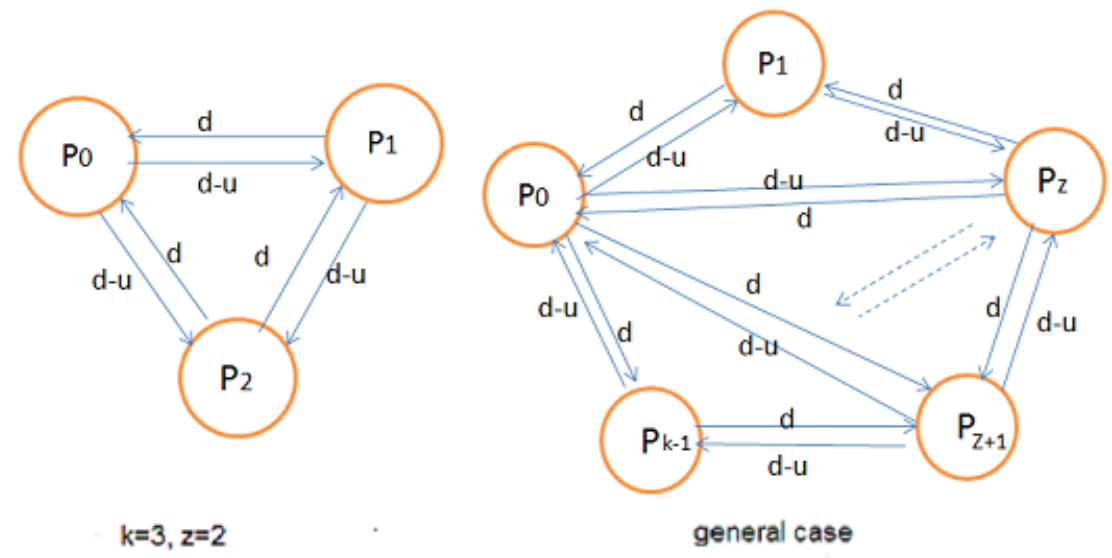

Fig. 13. Message Delays in $R_{2}\left(R_{2}^{\prime}\right)$

- For $k \leq l \leq n-1,0 \leq l^{\prime} \leq n-1, d_{l, l^{\prime}}=d-u / 2-x_{l}+x_{l}^{\prime}=d-u / 2+x_{l}^{\prime}$ (because $x_{l}=0$ ). $p_{l}^{\prime}$ is shifted by $x_{l}^{\prime}$ where $\left|x_{l}^{\prime}\right|<u / 2$, so $d-u \leq d_{l, l^{\prime}} \leq d$. In the same way, we can prove $d-u \leq d_{l^{\prime}, l} \leq d$.

Step 2.1: By the definition of the deterministic object and the assumed correctness of the algorithm, there exists at least one legal permutation $\pi^{\prime}$ for the $k$ operations in $R_{2}$, such that for any operation sequence $\rho^{*}$, if $\rho \circ \pi^{\prime} \circ \rho^{*}$ is legal, then when the operations in $\rho^{*}$ are invoked sequentially after $t+u$ (after all the operations in $R_{2}$ return), each operation returns the same value as in $\rho^{*}$.

Step 2.2: We show $o p_{z}$ cannot be the last operation in $\pi^{\prime}$. Because $p_{z}$ is shifted by $-[(k-1) / 2 k] \cdot u$ and $p_{(z+1) \% k}$ is shifted by $[(k-1) / 2 k] \cdot u, o p_{(z+1) \% k}$ is invoked after $o p_{z}$ returns. $([(k-1) / 2 k-(-(k-1) / 2 k)] \cdot u=[(k-1) / k] \cdot u>|O P|)$. So in any permutation $\pi^{\prime}$ that respects the real time sequence in $R_{2}, o p_{z}$ cannot be the last operation.

Step 3: From the two steps above we have last $(\pi) \neq \operatorname{last}\left(\pi^{\prime}\right)$. According to the assumptions in the theorem, because $\operatorname{last}(\pi) \neq \operatorname{last}\left(\pi^{\prime}\right)$, there exists an operation 


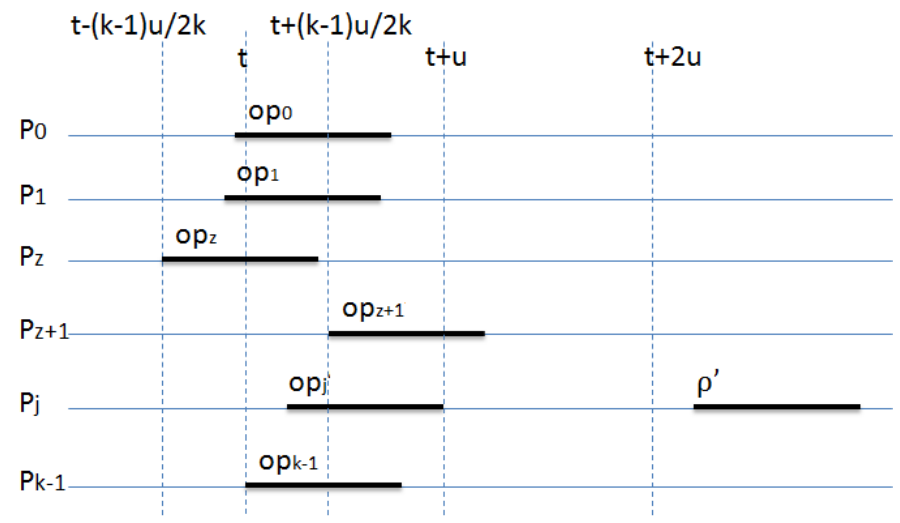

Fig. 14. Run $R_{2}\left(R_{2}^{\prime}\right)$

sequence $\rho^{\prime}$ such that one of the below is legal while the other is illegal:

(1) $\rho \circ \pi \circ \rho^{\prime}$

(2) $\rho \circ \pi^{\prime} \circ \rho^{\prime}$

Without loss of generality, assume $\rho \circ \pi \circ \rho^{\prime}$ is legal and thus $\rho \circ \pi^{\prime} \circ \rho^{\prime}$ is illegal. Let $R_{1}$ be extended to $R_{1}^{\prime}$ where a process invokes operations in $\rho^{\prime}$ sequentially after $t+2 u$. And let $R_{2}^{\prime}=\operatorname{shift}\left(R_{1}^{\prime}, \vec{x}\right)$. Then it is easy to prove that $R_{2}^{\prime}$ is an extension of $R_{2}$, because the shift amounts from $R_{1}$ to $R_{2}$ and from $R_{1}^{\prime}$ to $R_{2}^{\prime}$ are the same. $R_{2}^{\prime}$ is admissible but $\rho \circ \pi^{\prime} \circ \rho^{\prime}$ is illegal, contradicting the assumed correctness of the algorithm.

Step 4: By repeating Step 3 for every $\pi^{\prime}$ which satisfies the condition in Step 2.1 , we can prove there is a contradiction for every $\pi^{\prime}$, which means $\pi^{\prime}$ does not exist, and so $o p_{z}$ cannot be the last operation in $\pi$. By repeating Step 2 - Step 3 for every $\pi$ which satisfies the condition in Step 1.1, we can prove none of the $k$ operations can be the last one in $\pi$, which means $\pi$ does not exist, contradicting the assumed correctness of the algorithm. 
This lower bound can be applied to the message passing system with $n$ processes where $n$ is a finite integer. If $k=2$, then $(1-1 / k) u=u / 2$, matching the time lower bound proof for non-self-commuting property, also matching that non-self-commuting is one special case of non-self-permuting. And for the write, enqueue and push operation, we know that $k=n$. So the lower bound for them is $(1-1 / n) u$, matching the optimal local clock skew.

\section{E. Immediately Non-commuting Pairs of Operation Types}

For any two operation types $O P_{1}$ and $O P_{2}$ which immediately do not commute, $\left|O P_{1}\right|+\left|O P_{2}\right| \geq d[3]$. In the perfect synchronous system, it has been proved to be a tight time bound for immediately non-commuting pure mutators and pure accessors [3]. In the partially synchronous system, the lower bound still holds.

Compared with the eventually self-commuting operation types, we are more interested in eventually non-self-commuting operation types, because usually an object has several operation types and not all pairs of two pure mutators are eventually commuting. Suppose two operation types $O P_{1}$ and $O P_{2}$ eventually do not commute with each other and $O P$ is an eventually non-self-commuting operation type. Then having two concurrent operations $o p_{1} \in O P_{1}$ and $o p_{2} \in O P_{2}$ is similar to the case of having two concurrent operations $o p_{1}, o p_{2} \in O P$. By solving the linearizability problem for the eventually non-self-commuting operations, the problem for a pair of eventually non-commuting operations can also be solved.

Mavronicolas and Roth [7] prove for write and read on a shared object that the total time lower bound is at least $d+\min \{\epsilon, u\} / 2$. However, this result only applies for certain class of algorithms with the constraints - object symmetric, which means each process handles activity involving a certain object in precisely the same way it 
handles activity on any other. We want to get rid of that constraint in our work.

Below we prove $\left|O P_{1}\right|+\left|O P_{2}\right| \geq d+\min \{\epsilon, u, d / 3\}$ where $O P_{1}$ is immediately self-commuting but eventually non-self-commuting and non-overwriting, and $\mathrm{OP}_{2}$ is a pure mutator which immediately does not commute with $O P_{1}$. The theorem can be applied to push and peek on a stack, and enqueue and peek on a queue. However, it cannot be applied to the write and read operations on a register.

We let $O P_{1}$ be immediately self-commuting, because if $O P_{1}$ is immediately nonself commuting, the lower bound has been proved to be $d+\min \{\epsilon, u, d / 3\}$ (Theorem C.1), which is the same as the lower bound we prove below.

We let $O P_{2}$ be a pure accessor (recall that a pure accessor does not modify the object) because:

1. If $O P_{2}$ modifies the object, it can be an overwriter or it can reverse the modifications of $O P_{1}$. Then $O P_{2}$ has the same effect as an overwriter. Although we can add constraints on $\mathrm{OP}_{2}$ to exclude these cases, the theorem itself will be too complicated.

2. For the commonly used pair of immediately non-commuting operations such as push and pop, enqueue and dequeue, we have already proved that the lower bound for pop and dequeue is $d+\min \{\epsilon, u, d / 3\}$ (Theorem C.1), which is the same as in the following proof.

Under these assumptions of $O P_{1}$ and $O P_{2}$, we use two operations $o p_{1} \in O P_{1}$ and $o p_{1}^{\prime} \in O P_{1}$. There are three ways to see if $o p_{1}$ should be put before $o p_{1}^{\prime}$ in the legal permutation of concurrent operations:

1. $o p_{1}$ completes before $o p_{1}^{\prime}$ is invoked. 
2. An operation $o p_{2} \in O P_{2}$ invoked after $o p_{1}$ and $o p_{1}^{\prime}$ complete can tell which one should be arranged as the first operation.

3. An operation $o p_{3} \in O P_{2}$ that reflects the modification of $o p_{1}$ has been completed on the object while the modifications of $o p_{1}^{\prime}$ are not executed.

We can construct counter-examples to prove the violation of linearizability by using the three points above. For example, we can let $o p_{1}^{\prime}$ be invoked after $o p_{1}$ completes, then let another operation $o p_{2}$ be invoked after $o p_{1}^{\prime}$ completes. If the return value of $o p_{2}$ reflects that $o p_{1}^{\prime}$ should be arranged before $o p_{1}$, then there is a contradiction.

However, the third point has a problem. Suppose $O P_{1}$ is the write operation on a read/write register and $O P_{2}$ is the read operation on the read/write register. If the register is initialized with zero, and write(1), write(2) and read(2) are concurrent operations, then there are two legal permutations - write $(2) \circ \operatorname{read}(2) \circ$ write $(1)$ and write $(1) \circ$ write $(2) \circ \operatorname{read}(2)$. So if $O P_{1}$ can overwrite the whole state of the object, even if $o p_{3}$ reflects that $o p_{1}$ has been completed, it cannot tell whether $o p_{1}^{\prime}$ has not been executed or $o p_{1}^{\prime}$ has been executed but overwritten by $o p_{1}$. That is why the non-overwriting property may impact the time lower bound.

Theorem E.1 For immediately self-commuting operation type $O P$ and pure accessor $A O P$, if there exist an operation sequence $\rho$, operation $o p_{1}, o p_{2} \in O P$ and $a o p_{1}, \operatorname{aop}_{2}, \operatorname{aop}_{3} \in A O P$ such that:

A: one of the below is legal while the other one is illegal:

- $\rho \circ o p_{1} \circ a o p_{1}$

- $\rho \circ o p_{2} \circ o p_{1} \circ a o p_{1}$

B: one of the below is legal while the other one is illegal: 
- $\rho \circ o p_{2} \circ a \circ p_{2}$

- $\rho \circ o p_{1} \circ o p_{2} \circ a o p_{2}$

and $C$ : one of the below is legal while the other one is illegal:

- $\rho \circ o p_{1} \circ o p_{2} \circ a o p_{3}$

- $\rho \circ o p_{2} \circ o p_{1} \circ a o p_{3}$

then $|O P|+|A O P| \geq d+\min \{\epsilon, u, d / 3\}$ in an $n$-process $(n \geq 3)$ message passing system with message delay bound $[d-u, d]$ and local clock skew bound $\epsilon$.

Proof. Assume in contradiction that there are two operation types $O P$ and $A O P$ which satisfy the properties in the theorem but $|O P|+|A O P|<d+m$ time where $m=$ $\min \{\epsilon, u, d / 3\}$. According to the theorem statement, we assume $o p_{1}=O P\left(\arg _{1}\right.$, ret $\left._{1}\right)$, $o p_{2}=O P\left(\arg _{2}\right.$, ret $\left._{2}\right)$ and $a o p_{1}=A O P\left(\operatorname{aarg}_{1}, \operatorname{aret}_{1}\right), \operatorname{aop}_{2}=A O P\left(\operatorname{aarg}_{2}, \operatorname{aret}_{2}\right)$, $\operatorname{aop}_{3}=A O P\left(\operatorname{aarg}_{3}, \operatorname{aret}_{3}\right)$.

Because either $\rho \circ o p_{1} \circ o p_{2} \circ a o p_{3}$ or $\rho \circ o p_{2} \circ o p_{1} \circ a o p_{3}$ is legal, at least one of $\rho \circ o p_{1} \circ o p_{2}$ and $\rho \circ o p_{2} \circ o p_{1}$ is legal. If $\rho \circ o p_{1} \circ o p_{2}$ is legal, $\rho \circ o p_{2} \circ o p_{1}$ is also legal, because $o p_{1}$ and $o p_{2}$ are immediately self-commuting operations. Vice versa. So we have both of the below are legal:

- $\rho \circ o p_{1} \circ o p_{2}$

- $\rho \circ o p_{2} \circ o p_{1}$

The counter-example is shown as below. (Similarly to theorem C.1, we let one process invokes $\rho$ in sequence and wait until the system becomes quiescent for a long enough time. And we ignore $\rho$ in the following runs.) Proof structure refers to Fig. 15.

Step 1: We construct run $R_{1}$ as follows (Fig. 16). Let $p_{i}, p_{j}$ and $p_{k}$ be three specific processes and $p_{l}$ denotes any other process: 


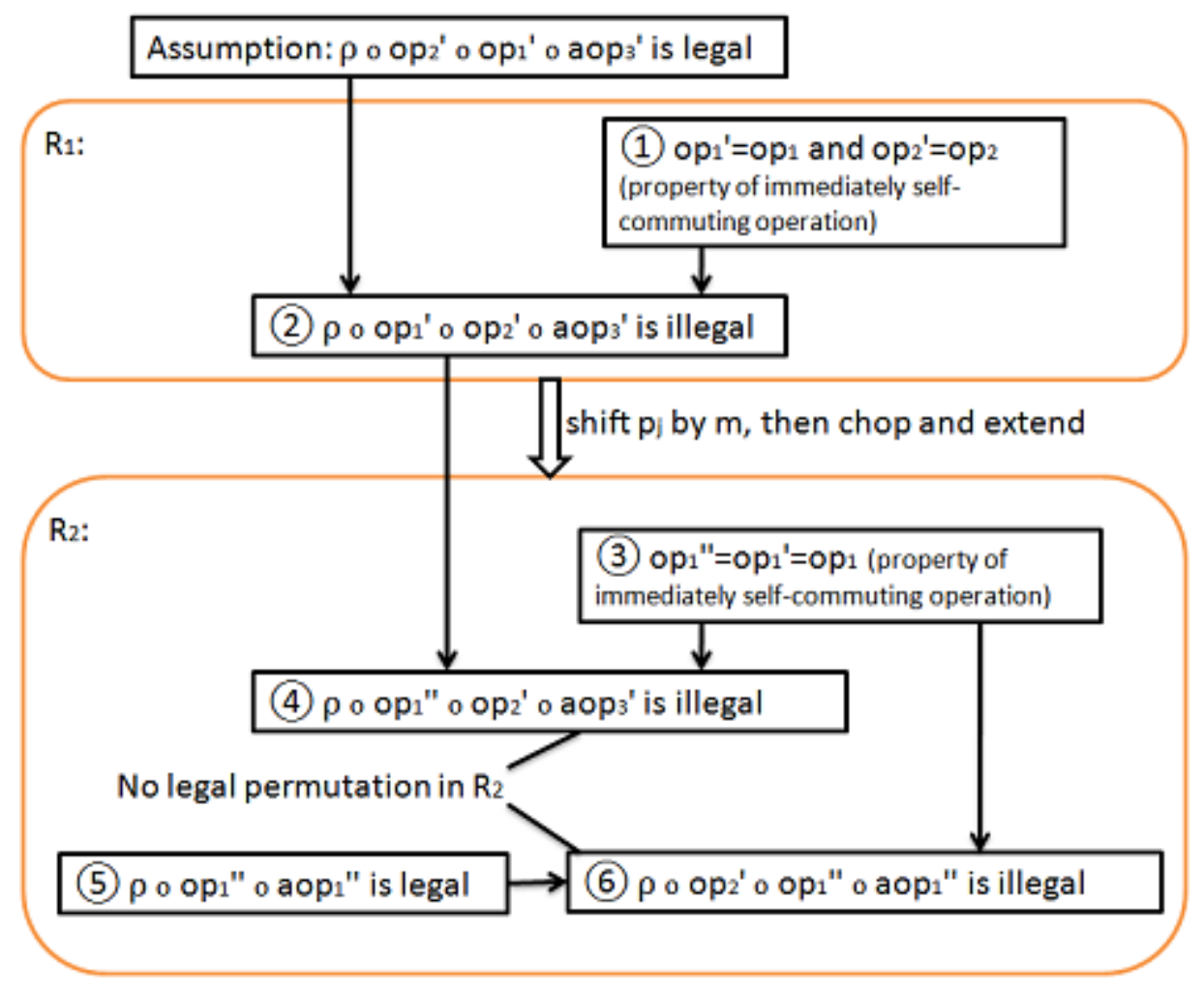

Fig. 15. Proof Structure of Theorem E.1

- All the processes have the same local clock function.

- $d_{i, k}=d_{i, l}=d_{j, k}=d_{j, l}=d$, and $d_{i, j}=d_{j, i}=d_{k, i}=d_{l, i}=d_{k, j}=d_{l, j}=d-m$. $d_{k, l}$ and $d_{l, k}$ is any value in the range $[d-u, d]$.

$R_{1}$ is admissible because:

1. the maximum local clock skew is $0<\epsilon$; and

2. letting the message delay be $d-m$ or $d$ is admissible.

In run $R_{1}, p_{i}$ executes $o p_{1}^{\prime} \in O P$ with $\arg _{1}$ invoked at time $t ; p_{j}$ executes $o p_{2}^{\prime} \in$ $O P$ with $\arg _{2}$ invoked at time $t$. Suppose $o p_{1}^{\prime}$ returns at real time $t_{1}$ and $o p_{2}^{\prime}$ returns at real time $t_{2}$. Let $t_{\max }=\max \left\{t_{1}, t_{2}\right\}$. Then $p_{i}$ executes $a o p_{1}^{\prime} \in A O P$ with $a a r g_{1}$ 
invoked at real time $t_{\max } . p_{j}$ executes $a p_{2}^{\prime} \in A O P$ with $\operatorname{aarg}_{2}$ invoked at $t_{\max }$. Process $p_{k}$ executes $a o p_{3}^{\prime} \in A O P$ with $\operatorname{aarg}_{3}$ invoked at real time $t_{\text {max }}+m$. No other operations are invoked.

Since $a o p_{1}^{\prime}, a o p_{2}^{\prime}$ and $a o p_{3}^{\prime}$ are invoked after $o p_{1}^{\prime}$ and $o p_{2}^{\prime}$ return, in any legal permutation of the five operations, $a o p_{1}^{\prime}, a o p_{2}^{\prime}$ and $a o p_{3}^{\prime}$ must be put after $o p_{1}^{\prime}$ and $o p_{2}^{\prime}$. Because $|O P|+|A O P|<d+m, a o p_{1}^{\prime}$ and $a o p_{2}^{\prime}$ return before $t+d+m$ and $a o p_{3}^{\prime}$ returns before $t+d+2 m$. Since $a o p_{1}^{\prime}$ and $a o p_{2}^{\prime}$ are pure mutators, it doesn't matter if they are before or after $a p_{3}^{\prime}$ in the linearized permutation. Therefore, by the assumed correctness of the algorithm, at least one of $\rho \circ o p_{1}^{\prime} \circ o p_{2}^{\prime} \circ a \circ p_{3}^{\prime}$ and $\rho \circ o p_{2}^{\prime} \circ o p_{1}^{\prime} \circ a o p_{3}^{\prime}$ must be legal. Without loss of generality we assume $\rho \circ o p_{2}^{\prime} \circ o p_{1}^{\prime} \circ a \circ p_{3}^{\prime}$ is legal.

Step 1.1: First we show $o p_{1}^{\prime}=o p_{1}$ and $o p_{2}^{\prime}=o p_{2}$.

Because $o p_{1}^{\prime}$ and $o p_{2}^{\prime}$ are before $a o p_{1}^{\prime}, a o p_{2}^{\prime}$ and $a o p_{3}^{\prime}$ in any legal permutation, the assumed correctness of the algorithm ensures at least one of the below is legal:

- $\rho \circ o p_{1}^{\prime} \circ o p_{2}^{\prime}$

- $\rho \circ o p_{2}^{\prime} \circ o p_{1}^{\prime}$

If $\rho \circ o p_{1}^{\prime} \circ o p_{2}^{\prime}$ is legal, by the definition of the deterministic object, because $\rho \circ o p_{1} \circ o p_{2}$ is legal, $o p_{1}^{\prime}=o p_{1}$. Then because of the same reason, $o p_{2}^{\prime}=o p_{2}$. If $\rho \circ o p_{2}^{\prime} \circ o p_{1}^{\prime}$ is legal, we can get the same conclusion in the similar way.

Step 1.2: Now we prove $\rho \circ o p_{1}^{\prime} \circ o p_{2}^{\prime} \circ a \circ p_{3}^{\prime}$ must be illegal by using assumption $\mathrm{C}$ in the theorem with the case analysis below(2) in Fig. 15).

Case 1: $\rho \circ o p_{2} \circ o p_{1} \circ a o p_{3}$ is legal and $\rho \circ o p_{1} \circ o p_{2} \circ a \circ p_{3}$ is illegal.

Because $o p_{1}^{\prime}=o p_{1}, o p_{2}^{\prime}=o p_{2}$, and $\rho \circ o p_{2}^{\prime} \circ o p_{1}^{\prime} \circ a o p_{3}^{\prime}$ is legal, by the definition of the deterministic object, we get $a o p_{3}^{\prime}=a \circ p_{3}$. Therefore $\rho \circ o p_{1}^{\prime} \circ o p_{2}^{\prime} \circ a \circ p_{3}^{\prime}$ is illegal because $\rho \circ o p_{1} \circ o p_{2} \circ a o p_{3}$ is illegal.

Case 2: $\rho \circ o p_{2} \circ o p_{1} \circ a o p_{3}$ is illegal and $\rho \circ o p_{1} \circ o p_{2} \circ a o p_{3}$ is legal. 
Because $o p_{1}^{\prime}=o p_{1}, o p_{2}^{\prime}=o p_{2}$, and $\rho \circ o p_{2}^{\prime} \circ o p_{1}^{\prime} \circ a o p_{3}^{\prime}$ is legal, by the definition of the deterministic object, we get $a \circ p_{3}^{\prime} \neq a \circ p_{3}$. Therefore $\rho \circ o p_{1}^{\prime} \circ o p_{2}^{\prime} \circ a \circ p_{3}^{\prime}$ is illegal because $\rho \circ o p_{1} \circ o p_{2} \circ a o p_{3}$ is legal.

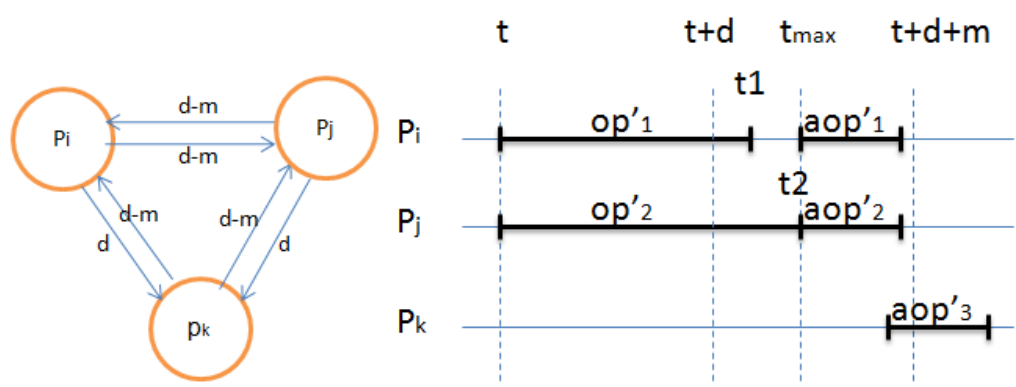

(a) Message Delays in $R_{1}$

(b) $R_{1}$

Fig. 16. Step 1 in Theorem E.1

Step 2: Define run $R_{2}^{\prime}=\operatorname{shift}\left(R_{1}, \vec{x}\right)$, where $x_{j}=m$ and $x_{l}=0$ for all $l \neq j$, which means $p_{i}$ executes $o p_{1}^{\prime}$ with $\arg _{1}$ invoked at real time $t, p_{j}$ execute $o p_{2}^{\prime}$ with $\arg _{2}$ invoked at time $t+m, a o p_{1}^{\prime}$ and $a o p_{3}^{\prime}$ are still executed at the same real time as in $R_{1}$, and $a o p_{2}^{\prime}$ is executes $m$ real time later than in $R_{1}$ (refer to Fig. 17).

Now we apply Lemma B.1 here. According to formula (1), $d_{j, i}^{\prime}$ is $d-2 m$ which may violate the message delay constraint, and it is the only possible invalid message delay. The earliest possible message from $p_{j}$ to $p_{i}$ is sent no earlier than $t+m$, because (1) $o p_{2}^{\prime}$ is invoked at $t+m$ in $R_{2}^{\prime}$; (2) the earliest message in $R_{2}^{\prime}$ is sent after $t$, and so if it is received by $p_{j}$, it is received after $t+d-m>t+m$. Let $R_{2}^{\prime \prime}=\operatorname{chop}\left(R_{2}^{\prime}, d-m\right)$. In the chopping procedure, $t^{*}=t+m+\min \{d-2 m, d-m\}=t+d-m$. Вy Lemma B.1, $R_{2}^{\prime \prime}$ is admissible.

$V_{i} \in R_{2}^{\prime \prime}$ ends just before $t+d-m$. So the invocation of $o p_{1}^{\prime}$ occurs in $R_{2}^{\prime \prime}$ but the response may not. For process $p_{j}$, because $d_{i, j}^{\prime}=(d-m)-0+m=d$ and 
$d_{i, k}^{\prime}=d_{i, l}^{\prime}=d$, we get $D_{i, j}^{\prime}=d$. So $V_{j} \in R_{2}^{\prime \prime}$ ends just before $t+d-m+d \geq t+d+2 m$ (because $m \leq d / 3$ ). Because $a \circ p_{2}^{\prime}$ returns before $t+d+m$ in $R_{1}$, we get $a o p_{2}^{\prime}$ returns before $t+d+2 m$ in $R_{2}^{\prime}$. So $V_{j}$ ends after $a p_{2}^{\prime}$ returns. Similarly, we can prove $V_{k} \in R_{2}^{\prime \prime}$ ends after $a o p_{3}^{\prime}$ returns.

We can extend $R_{2}^{\prime \prime}$ to a complete admissible run $R_{2}$ by setting the delay for messages from $p_{j}$ to $p_{i}$ to be $d>\delta$. The response of $o p_{1}^{\prime}$ may not occur in $V_{i}$ and it may be different from ret $_{1}^{\prime}$. So we call the operation invoked by $p_{i}$ at real time $t$ $o p_{1}^{\prime \prime}$. In $R_{2}$, if the invocation of $a o p_{1}^{\prime}$ is not in $R_{2}^{\prime \prime}$ (meaning $t+d-m<t_{\max }$ ), let $p_{i}$ invoke operation $a o p_{1}^{\prime \prime} \in A O P$ with $\operatorname{aarg}_{1}$ after $o p_{1}^{\prime \prime}$ returns and $V_{i}$ ends. Depending on whether $o p_{1}^{\prime \prime}$ returns later or $V_{i}$ ends later, $a o p_{1}^{\prime \prime}$ is invoked immediately after the later one of them occurs. So $a p_{1}^{\prime \prime}$ is invoked at $\max \left\{\min \left\{t_{\max }, t+d-m\right\}, t+\left|o p_{1}^{\prime \prime}\right|\right\}$. Here $t+d-m$ is the ending time of $V_{i}$, and $t+\left|o p_{1}^{\prime \prime}\right|$ is the response time of operation $o p_{1}^{\prime \prime}$.

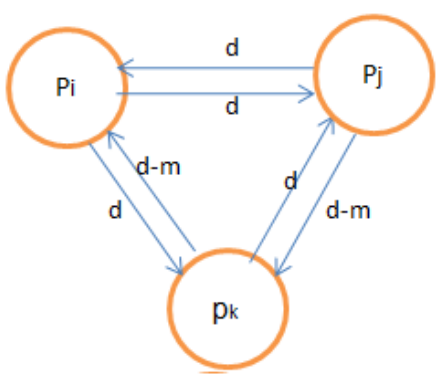

(a) Message Delays in $R_{2}$

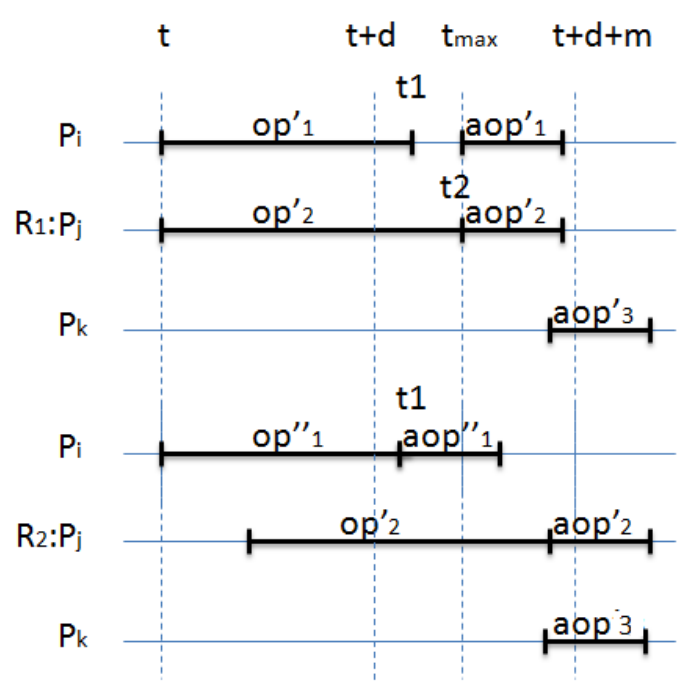

(b) $R_{1}$ and $R_{2}$

Fig. 17. Step 2 in Theorem E.1 
Step 2.1: First we show $o p_{1}^{\prime \prime}=o p_{1}$ (3) in Fig. 15) and thus $\rho \circ o p_{1}^{\prime \prime} \circ o p_{2}^{\prime} \circ a o p_{3}^{\prime}$ is illegal (4) in Fig. 15).

By the assumed correctness of the algorithm, at least one of $\rho \circ o p_{1}^{\prime \prime} \circ o p_{2}$ and $\rho \circ o p_{2} \circ o p_{1}^{\prime \prime}$ is legal. No matter which one is legal, since we have that $\rho \circ o p_{1} \circ o p_{2}$ and $\rho \circ o p_{2} \circ o p_{1}$ are both legal, by the definition of the deterministic object, it follows that $o p_{1}^{\prime \prime}=o p_{1}$. And since in Step 1.2 we have proved $\rho \circ o p_{1}^{\prime} \circ o p_{2}^{\prime} \circ a \circ p_{3}^{\prime}$ is illegal, $\rho \circ o p_{1}^{\prime \prime} \circ o p_{2}^{\prime} \circ a \circ p_{3}^{\prime}$ is illegal.

Step 2.2: We show $\rho \circ o p_{2}^{\prime} \circ o p_{1}^{\prime \prime} \circ a \circ p_{1}^{\prime \prime}$ is illegal(6) in Fig. 15).

Step 2.2a: First we prove $a o p_{1}^{\prime \prime}$ returns before $t+d+m$. The invocation time of $a o p_{1}^{\prime \prime}$ is $\max \left\{\min \left\{t_{\max }, t+d-m\right\}, t+\left|o p_{1}^{\prime \prime}\right|\right\}$.

1. Case 1: $t_{\max } \leq t+d-m$. Then $a p_{1}^{\prime \prime}$ is invoked before $t+d-m$ and $o p_{1}^{\prime \prime}$ responds before $t+d-m$, meaning $t+\left|o p_{1}^{\prime \prime}\right|<t+d-m$. Therefore, aop $p_{1}^{\prime \prime}$ is invoked at $t_{\max }$.

(a) Case 1.1: $t_{\max }=t_{1}$. Then $a o p_{1}^{\prime \prime}$ is invoked immediately after $o p_{1}^{\prime \prime}$ responds. Because $\left|o p_{1}^{\prime \prime}\right|+\left|a o p_{1}^{\prime \prime}\right|<d+m$ and $o p_{1}^{\prime \prime}$ is invoked at real time $t, a o p_{1}^{\prime \prime}$ returns before $t+d+m$.

(b) Case 1.2: $t_{\max }=t_{2}$. Then $\left|o p_{2}^{\prime}\right|=t_{\max }-t$. If $a o p_{1}^{\prime \prime}$ does not return before $t+d+m$, then $\left|o p_{2}^{\prime}\right|+\left|a o p_{1}^{\prime \prime}\right| \geq\left(t_{\max }-t\right)+\left(t+d+m-t_{\max }\right)=d+m$, contradicting that $|O P|+|A O P|<d+m$.

2. Case 2: $t+d-m<t_{\max }$ and $t+d-m \leq t+\left|o p_{1}^{\prime \prime}\right|$. Then $a o p_{1}^{\prime \prime}$ is invoked at $t+\left|o p_{1}^{\prime \prime}\right|$, immediately after $o p_{1}^{\prime \prime}$ responds. Similarly to case 1.1 , $a o p_{1}^{\prime \prime}$ returns before $t+d+m$.

3. Case 3: $t+d-m<t_{\max }$ and $t+d-m>t+\left|o p_{1}^{\prime \prime}\right|$. Then $a p_{1}^{\prime \prime}$ is invoked at $t+d-m$. 
(a) Case 3.1: $t_{\max }=t_{1}$. Then $o p_{1}^{\prime}$ returns at $t_{\max }>t+d-m$. So $o p_{1}^{\prime \prime}$ cannot return before $t+d-m$, because until $t+d-m, p_{i}$ has the same local view in $R_{1}$ and $R_{2}$. This contradicts $t+d-m>t+\left|o p_{1}^{\prime \prime}\right|$.

(b) Case 3.2: $t_{\max }=t_{2}$. Similarly to case 1.2, if $a \circ p_{1}^{\prime \prime}$ does not return before $t+d+m$, we can get $\left|o p_{2}^{\prime}\right|+\left|a o p_{1}^{\prime \prime}\right| \geq\left(t_{\max }-t\right)+(t+d+m-(t+d-m))=$ $t_{\text {max }}-t+2 m>(t+d-m)-t+2 m>d+m$, contradicting that $|O P|+|A O P|<d+m$.

Step 2.2b: Now we analyze $R_{2}$ from the view of $p_{i}$. Because $o p_{2}^{\prime \prime}$ is invoked at $t+m$ and $D_{j, i}^{\prime}=d$, it is impossible for $p_{i}$ to get any information about $o p_{2}^{\prime \prime}$ before $t+d+m$, which is after $a p_{1}^{\prime \prime}$ returns. So without learning the existence of $o p_{2}^{\prime \prime}, p_{i}$ returns $a o p_{1}^{\prime \prime}$ such that $\rho \circ o p_{1}^{\prime \prime} \circ a o p_{1}^{\prime \prime}$ is legal. Because $o p_{1}^{\prime \prime}=o p_{1}, o p_{2}^{\prime}=o p_{2}$ and $\rho \circ o p_{1}^{\prime \prime} \circ a \circ p_{1}^{\prime \prime}$ is legal, similarly to the analysis in step 1.2, by using assumption A in the theorem, $\rho \circ o p_{2}^{\prime} \circ o p_{1}^{\prime \prime} \circ a o p_{1}^{\prime \prime}$ is illegal.

Contradiction: From step 2.1, because $\rho \circ o p_{1}^{\prime \prime} \circ o p_{2}^{\prime} \circ a \circ p_{3}^{\prime}$ is illegal and $a o p_{3}^{\prime}$ is invoked after $o p_{2}^{\prime}$ returns, by the assumed correctness of the algorithm, in any legal permutation of the operations in $R_{2}, o p_{1}^{\prime \prime}$ must be put after $o p_{2}^{\prime}$. From step 2.2, because $\rho \circ o p_{2}^{\prime} \circ o p_{1}^{\prime \prime} \circ a \circ p_{1}^{\prime \prime}$ is illegal and $a o p_{1}^{\prime \prime}$ is invoked after $o p_{1}^{\prime \prime}$ returns, by the assumed correctness of the algorithm, in any legal permutation of the operations in $R_{2}, o p_{2}^{\prime}$ is after $o p_{1}^{\prime \prime}$, contradicting the conclusion from step 2.1 that $o p_{1}^{\prime \prime}$ must be put after $o p_{2}^{\prime}$. Therefore, $R_{2}$ is an admissible run but there is no legal permutation for the operations in $R_{2}$, contradicting the assumed correctness of the algorithm.

Remark:

1. In theorem E.1, although we never explicitly say $O P$ and $A O P$ immediately do not commute with each other, they are immediately non-commuting operations. The explanation is as below. 
Suppose in contradiction, $O P$ and $A O P$ are immediately commuting operations. From the assumption in theorem E.1, there exist instances $o p_{1}, o p_{2}$ of $O P$, and instance $a_{0} p_{3}$ of $A O P$, such that one of the below is legal while the other one is illegal:

(1) $\rho \circ o p_{1} \circ o p_{2} \circ a \circ p_{3}$

(2) $\rho \circ o p_{2} \circ o p_{1} \circ a \circ p_{3}$

Without loss of generality, let's assume $\rho \circ o p_{1} \circ o p_{2} \circ a o p_{3}$ is legal. Since $O P$ and $A O P$ are immediately commuting, we can switch $o p_{2}$ and $a o p_{3}$ and get $\rho \circ o p_{1} \circ a \circ p_{3} \circ o p_{2}$ is legal. In a similar way, we can continue to switch $o p_{1}$ and $a o p_{3}$, without affecting $o p_{2}$ because $a \mathrm{op}_{3}$ is a pure accessor, and we get $\rho \circ a \circ p_{3} \circ o p_{1} \circ o p_{2}$ is legal. Next, because $o p_{1}$ and $o p_{2}$ are immediately commuting, we exchange the position of them and get $\rho \circ a \circ p_{3} \circ o p_{2} \circ o p_{1}$ is legal. Finally, we switch $a o p_{3}$ back to be the last operation and get $\rho \circ o p_{2} \circ o p_{1} \circ a o p_{3}$ is legal, contradicting the assumption in the theorem. Therefore, $O P$ and $A O P$ are immediately non-commuting operations.

2. In theorem E.1, we assumed $\rho \circ o p_{2}^{\prime} \circ o p_{1}^{\prime} \circ a \circ p_{3}^{\prime}$ is legal in Step 1, and then use assumptions $\mathrm{A}$ and $\mathrm{C}$ to get the contradiction in the counter-example. If $\rho \circ \circ p_{1}^{\prime} \circ \circ p_{2}^{\prime} \circ a \circ p_{3}^{\prime}$ is legal, then $p_{i}$ is shifted by $m$ and we will use assumptions $\mathrm{B}$ and $\mathrm{C}$ to get the contradiction. 


\section{CHAPTER V}

\section{UPPER BOUNDS FOR LINEARIZABLE OBJECTS}

In this chapter, we will present an implementation for an arbitrary data type with all types of operations. We group all the operations on a shared object into three types: pure accessors $A O P$, pure mutators $M O P$ and all the other operations $O O P$.

By a result in [6], the optimal value of $\epsilon$ is $(1-1 / n) u$, which is smaller than $u$. And there already exist some implementations for optimal $\epsilon$. The implementation for a shared object below is built on a message passing system whose clocks are synchronized to within the optimal $\epsilon$ and have no drift.

\section{A. Main Idea of the Implementation}

Each process keeps a copy of the object and we assume each copy is initialized with the same initial value of the object. The events in each process are triggered by operation invocation, message reception and timer expiration.

We start a timer with the function set_timer(counter, $\langle$ op, arg,ts $\rangle$, action). Each timer is set for an operation, so we add the information of the operation into the timer in the format $\langle o p, a r g, t s\rangle$, where $\arg$ is the argument of op, and $t s$ is the timestamp of op. Each timestamp is in the format $\langle$ clock_time,process_id $\rangle$ where clock_time is the local clock time and process_id is the id of op's invoking process. The timer counts down from the counter value to 0 . When it reaches 0 , the timer expires and expire_timer $(\langle o p, a r g, t s\rangle$, action) occurs. According to op and action, the timer expiration will trigger some events. Each timer can be canceled with the function cancel_timer $(\langle o p, a r g, t s\rangle$, action).

The local clock time of process $p_{i}$ is indicated by local_time ${ }_{i}$.

Execution of operation op with argument $\arg$ on the local copy of process $p_{i}$ is 
indicated by $\left(l_{o c a l} o b j_{i}, r e t\right)=$ execute_op $\left(l o c a l_{\_} o b j_{i}, o p, a r g\right) . \quad l o c a l_{-} o b j_{i}$ means the local copy held by process $p_{i}$. ret is the return value of op after the execution.

Process $p_{i}$ sends messages to all the other processes using the notation $\operatorname{sen}_{i}$. Process $p_{i}$ receives a message using the notation $r e c v_{i}$.

\section{1. $O O P$}

First we consider the operations belonging to $O O P$. We want these operations to be executed in each copy in the same order. This order is determined by the timestamps of operations.

Immediately after the operation invocation, we let the invoking process $p_{j}$ send the operation op $\in O O P$ with its argument and timestamp $\left\langle\right.$ local_time $\left._{j}, j\right\rangle$ together to all the other processes. Due to the local clock skew and message delay uncertainty, the order of operation receiving events may be different from the order of operations' timestamps. Each process $p_{i}$ uses a priority queue To_Execute $e_{i}$ to temporarily store each $\langle o p, a r g, t s\rangle$ that it receives and the priority queue uses $t s$ as the key.

To_Execute ${ }_{i}$ supports three operations: $\operatorname{add}(\langle o p, \arg , t s\rangle), \min ()$ and extract_min () . add $(\langle o p, a r g, t s\rangle)$ inserts $\langle o p, a r g, t s\rangle$ into To_Execute $e_{i}$. min () returns the minimum key in this queue. extract_min() returns the element with minimum key and removes it from the priority queue.

$p_{i}$ adds an operation into To_Execute E $_{i}$ immediately after receiving it. $p_{i}$ does not send messages to itself. So when $p_{i}$ sends $\langle o p$, arg,ts $\rangle$ to all the other processes, it starts a timer by set_timer $(d-u,\langle o p, a r g, t s\rangle, a d d)$ to count when to add $\langle o p, a r g, t s\rangle$ into its own priority queue. When this timer expires, it adds $\langle o p, a r g, t s\rangle$ into To_Execute ${ }_{i}$, pretending $\langle o p, a r g, t s\rangle$ is received through the fastest message, which is delivered $d-u$ time after it is sent.

After op is added into To_Execute ${ }_{i}, p_{i}$ waits at most $u+\epsilon$ time before execut- 
ing op on $p_{i}$ 's local copy. Because due to the message delay bound and local clock skew bound, after holding op in To_Execute E $_{i}$ for at most $u+\epsilon$ time, $p_{i}$ will not receive any operation whose timestamp is smaller than op (formal proof for this point comes after the pseudocode). The waiting time is controlled by another timer, which starts when op is added into To_Execute ${ }_{i}$ by set_timer $(u+\epsilon,\langle$ op, arg,ts $\rangle$, execute $)$. Once expire_timer $\left(\langle o p\right.$, arg, ts $\rangle$, execute) occurs, $p_{i}$ executes all the operations whose timestamps are no larger than op according to their timestamps sequence.

\section{2. $M O P$ and $A O P$}

Now, we think about pure mutators and pure accessors together. We define a parameter $X$ to be a value within $[0, d+\epsilon-u]$. The purpose of this time parameter is to regulate the trade-off of the response times between pure accessors and pure mutators, as in [7]. I.e., if pure accessors respond faster, then pure mutators respond slower, and vice versa.

The pure mutators modify the object. And different execution sequences of several pure mutators may result in different states of the object. For these operations, we still need them to be executed in the same order on each copy, otherwise later the operations may return some illegal value and violate the linearizability of the object. Similarly to the immediately non-self-commuting operations, we give them timestamps with the same mechanism, let their invoking processes send them to all the other processes and use To_Execute in each process to ensure the same order of their executions on the local copies of the object. Note here the pure mutators and all the other operations except pure accessors share the same To_Execute in each process, so in the pseudocode, we put the invocation of $M O P$ and $O O P$ together and use $o p$ to denote an operation which belongs to $M O P \cup O O P$. Because no two operations can be invoked by the same process at the same time, the timestamp of 
each operation is unique in To_Execute. So there is no problem to use the timestamps as keys in the priority queue.

However, because pure mutators do not return any information about the object, we can let them respond before they are executed on the local copies of processes. The bottom line is if they do not respond too fast, then the system can distinguish the order of two non-overlapping pure mutators. We let each pure mutator mop respond $\epsilon+X$ real time later after the invocation. It guarantees mop costs at least $\epsilon$ time, which is enough to distinguish the order of two non-overlapping pure mutators. The response time is counted by set_timer $(\epsilon+X,\langle$ mop, arg,ts $\rangle$, respond $)$. When this timer expires, the invoking process returns the acknowledgment of the pure mutator.

For the pure accessors, we do not need to broadcast them, because they do not modify the object. And we provide a different mechanism for their timestamps. For the timestamps of pure accesors, we use local invocation times minus $X$, pretending they are invoked $X$ time earlier. This would not affect the operation results of other concurrent operations, because pure accessors do not modify the object. The benefit is, if a pure accessor aop is invoked after a mutator op responds, aop must have a larger timestamp than op. And before aop returns, its invoking process executes op on the local copy. The execution and response time of aop is counted by set_timer $(d+$ $\epsilon-X,\langle a o p, a r g, t s\rangle$, respond $)$. When this timer expires, the invoking process executes all the operations in To_Execute whose timestamps are smaller than that of aop on its local copy of the object, and then execute aop.

Since the timestamp of a pure accessor is set to the local clock time minus $X$, is it possible that this timestamp is equal to the timestamp of another operation? No. In the later analysis, we will show this is impossible and the timestamp of each operation is unique among all the operations. 
B. Pseudocode

Now we display the pseudocode of this implementation in Algorithm 1.

Initialization in process $p_{i}:$ To_Execute $e_{i}=\emptyset$.

C. Correctness

\section{Summary of Observations}

Before we prove the correctness of this implementation, we summarize some observations from the pseudocode.

From lines 3-11, we get

Observation C.1 The earliest real time when each mutator $\langle o p, a r g, t s\rangle$ is added into any To_Execute ${ }_{i}$ (when set_timer $(u+\epsilon,\langle$ op, arg,ts $\rangle$, execute) occurs) is $d-u$ time after op is invoked.

Observation C.2 The latest real time when each mutator $\langle o p$, arg, ts $\rangle$ is added into any To_Execute $_{i}$ (when set_timer $(u+\epsilon,\langle$ op, arg,ts $\rangle$, execute) occurs) is d time after op is invoked.

From lines 12-20, we get

Observation C.3 When each mutator op is executed by any process $p_{i}$, all operations with smaller timestamps in To_Execute $e_{i}$ have been executed on local_obj . $_{\text {. }}$

From lines 21-28, we get

Observation C.4 When each pure accessor aop responds in $p_{i}$, all the operations in To_Execute T $_{i}$ with smaller timestamps than that of aop have been executed on local_obj . 


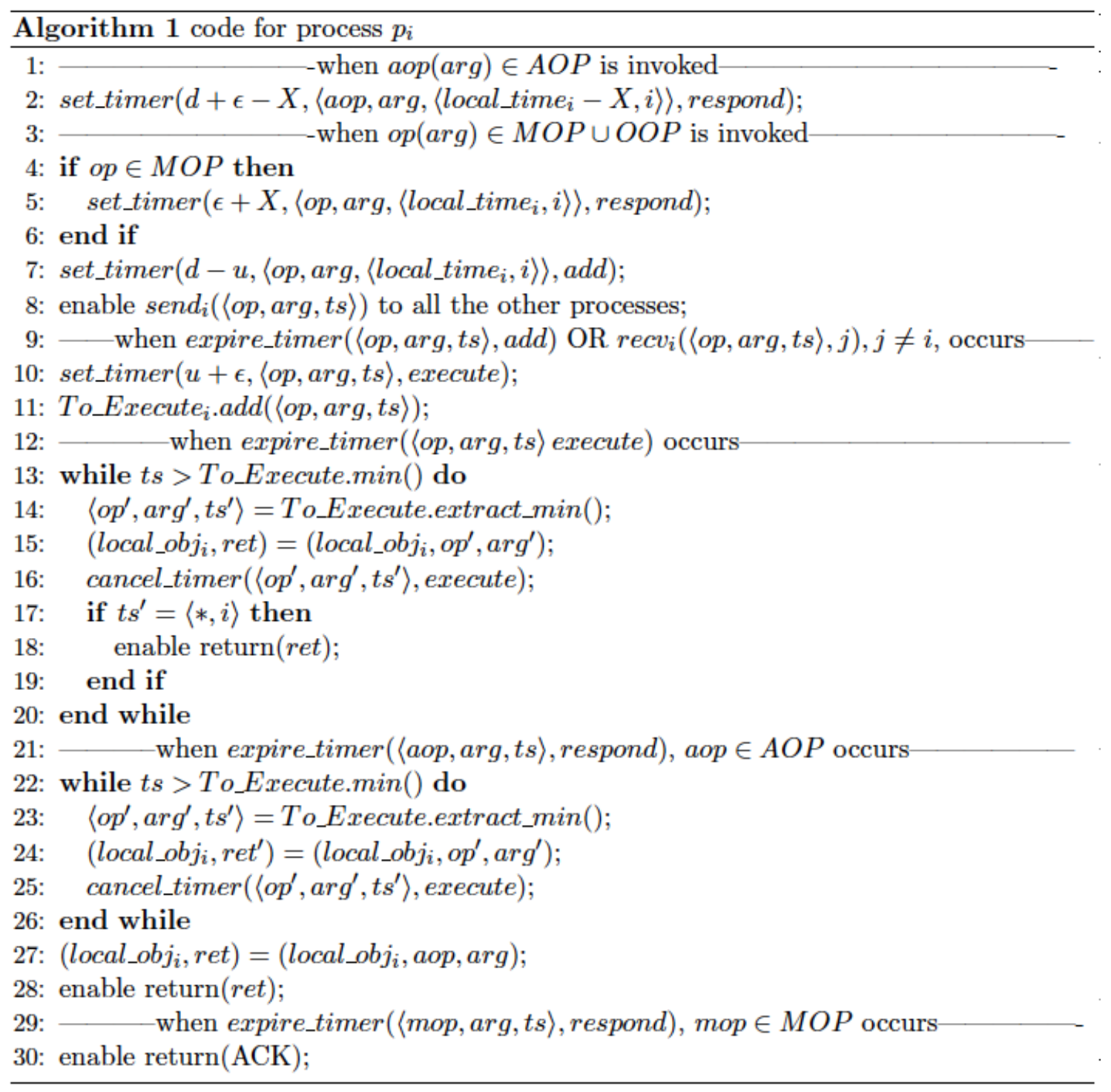


From lines 4-6 and 29-30, we get

Observation C.5 Each pure mutator responds $X+\epsilon$ real time after its invocation.

\section{Termination}

The termination of pure mutators is trivial from Observation C.5. Now we are going to prove the termination of $o o p \in O O P$ and pure accessors.

Lemma C.6 Each operation oop $\in$ OOP responds at most $d+\epsilon$ real time after its invocation.

Proof. Suppose oop is invoked by process $p_{i}$ at real time $t$. Then set_timer $(u+$ $\epsilon,\langle$ oop, arg,ts $\rangle$, execute) occurs at $t+d-u$ and expire_timer $(\langle o o p$, arg, ts $\rangle$, execute) occurs at $t+d-u+u+\epsilon=t+d+\epsilon$. At this time, if oop hasn't responded, the operations with timestamps smaller than oop in To_Execute E $_{i}$ will be executed one by one. After the executions of them, $p_{i}$ can execute oop on $l o c a l_{-} o b j_{i}$ and then oop responds. Since the local execution time is 0 , oop responds at most $d+\epsilon$ real time later after its invocation.

Now we prove the termination of each pure accessor.

Lemma C.7 Each pure accessor aop responds exactly $d+\epsilon-X$ time after its invocation.

Proof. Assume aop is invoked by process $p_{i}$. When expire_timer $(\langle a o p, a r g, t s\rangle$, respond) occurs (which is $d+\epsilon-X$ after the invocation 
of $a o p$ ), the only thing that can postpone the response of aop is that there exists some operation $\left\langle o p, a r g^{\prime}, t s^{\prime}\right\rangle$ in To_Execute $e_{i}$ and $t s^{\prime}<t s$. At this point, these operations with timestamps smaller than $t s$ will be executed one by one. After all the operations with smaller timestamps in To_Execute ${ }_{i}$ are executed, aop can be executed on $l_{o c a l} \_b j_{i}$ and then responds. Because the local computation time is 0, aop responds exactly $d+\epsilon-X$ time after its invocation.

\section{Linearizability}

We show the correctness of the implementation in three steps.

\section{a. $\quad$ Step I}

First we prove two important lemmas which will be used later.

Lemma C.8 After expire_timer $\left(\langle o p\right.$, arg,ts $\rangle$, execute) occurs in process $p_{i}$, no mutators with timestamps smaller than ts are added into To_Execute . $_{\text {. }}$

Proof. Assume, in contradiction that $\left\langle o p^{\prime}, a r g^{\prime}, t s^{\prime}\right\rangle$ is added into To_Execute $e_{i}$ after expire_timer $(\langle o p, a r g, t s\rangle$, execute $)$ and $t s^{\prime}<t s$. Assume op is invoked at real time t. By Observation C.1, the earliest time when set_timer $(u+\epsilon,\langle$ op, arg,ts $\rangle$, execute $)$ occurs at $p_{i}$ is $t+(d-u)$ and the earliest time when expire_timer $(\langle o p$, arg, ts $\rangle$, execute $)$ occurs at $p_{i}$ is $t+(d-u)+(u+\epsilon)=t+d+\epsilon$. So $\left\langle o p^{\prime}, \arg ^{\prime}, t s^{\prime}\right\rangle$ must be added into To_Execute $e_{i}$ after $t+d+\epsilon$. By Observation C.2, $o p^{\prime}$ is invoked after $t+d+\epsilon-d=t+\epsilon$. Because the local clock skew is bounded by $\epsilon$, if $o p^{\prime}$ is invoked more than $\epsilon$ time after $o p$ is invoked, $t s^{\prime}$ must be larger than $t s$, contradicting the assumption $t s^{\prime}<t s$. 
Lemma C.9 If process $p_{i}$ invokes a pure accessor aop with timestamp ts in $p_{i}$ at real time $t$, no operations with timestamps smaller than ts are added into To_Execute ${ }_{i}$ after $t+d+\epsilon-X$ (when expire_timer( $\langle a o p$, arg,ts $\rangle$, respond) occurs).

Proof. Assume, in contradiction that $\left\langle o p^{\prime}, a r g^{\prime}, t s^{\prime}\right\rangle$ is added into To_Execute T $_{i}$ after real time $t+d+\epsilon-X$ and $t s^{\prime}<t s$. Because the local clock skew upper bound is $\epsilon$ and since $t s$ uses $p_{i}$ 's local time at real time $t-X, o p^{\prime}$ must be invoked no later than $t-X+\epsilon$. Since every message delay is upper bounded by $d, p_{i}$ receives $\left\langle o p^{\prime}, a r g^{\prime}, t s^{\prime}\right\rangle$ no later than real time $t-X+\epsilon+d$ and also adds it into To_Execute E $_{i}$ no later than $t+d+\epsilon-X$, contradicting that $o p^{\prime}$ is added into To_Execute $e_{i}$ after $t+d+\epsilon-X$.

\section{b. Step II}

Then we use the two lemmas above to prove some lemmas for the real time sequence of different operation types, including mutator after mutator, pure accessor after pure accessor, pure accessor after mutator and mutator after pure accessor.

i. Mutator after Mutator

Lemma C.10 In every process $p_{i}$, all the mutators will be executed on local_obj $j_{i}$ in the sequence with their timestamps ascending.

Proof. When $p_{i}$ executes a mutator op on $l o c a l \_o b j_{i}$, by Observation C.3, all the mutators with smaller timestamps in To_Execute Th $_{i}$ have been executed; by Lemma C.8 and Lemma C.9, there is no any other operation with a smaller timestamp received by $p_{i}$ later. Since we have reliable message delivery between processes, when op is executed, all the operations with smaller timestamps have been received by $p_{i}$ and executed on $l o c a l \_o b j_{i}$. 
Lemma C.11 A mutator op (invoked by any process $p_{i}$ ) responds at least $\epsilon$ time after its invocation.

Proof. If $o p$ is a pure mutator, it responds exactly $\epsilon+X$ after its invocation. Since $X \geq 0$, it responds at least $\epsilon$ time after its invocation. If $o p$ is not a pure mutator, the response time depends on the execution times of the mutators

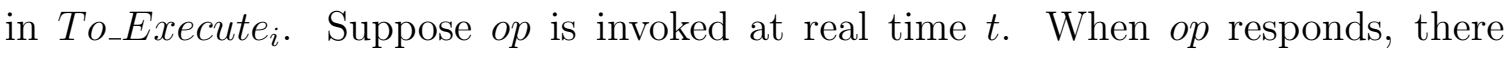
must exist a mutator $\left\langle o p^{\prime}, a r g^{\prime}, t s^{\prime}\right\rangle$ such that expire_timer $\left(\left\langle o p^{\prime}, a r g^{\prime}, t s^{\prime}\right\rangle\right.$, execute) occurs and $t s^{\prime} \geq t s$. By the bounded clock skew, $o p^{\prime}$ is invoked no earlier than $t-\epsilon$. By Observation C.1, set_timer $\left(u+\epsilon,\left\langle o p^{\prime}, a r g^{\prime}, t s^{\prime}\right\rangle\right.$, execute) occurs no earlier than $t-\epsilon+d-u$ and expire_timer $\left(\left\langle o p^{\prime}, a r g^{\prime}, t s^{\prime}\right\rangle\right.$, execute) occurs no earlier than $t-\epsilon+d-u+(u+\epsilon)=t+d$. So op responds no earlier than $t+d \geq t+\epsilon$.

Remark: From the proof above, we can answer the question mentioned before: Is it possible that another operation has the same timestamp as a pure accessor aop? The answer is NO. Because if such an operation op exists, it must be invoked by the same process as aop. And we can show:

- op $\notin A O P$. If op $\in A O P$, it must be invoked at the same time by the same process as aop, violating that there is only one pending operation in each process at any real time.

- op $\notin M O P$. If op $\in M O P$, since op and aop have the same timestamp, op is invoked $X$ time before the invocation of aop. But op responds exactly $\epsilon+X>$ $X$ time after its invocation. So when aop is invoked, op has not responded, violating that there is only one pending operation in each process at any real time. 
- op $\notin O O P$. If $o p \in O O P$, op responds at least $d$ time after its invocation (from the last sentence in the proof of Lemma C.11, which shows op is invoked at real time $t$ and responds no earlier than $t+d)$. Since $d>X(X \leq d+\epsilon-u$ and $\epsilon=(1-1 / n) u)$, similarly to the analysis for $M O P$, it violates that there is only one pending operation in each process at any real time.

From the analysis above, we can see that op does not exist, and so the answer of that question is NO.

Lemma C.12 If a mutator op $p_{1}$ responds before another mutator op $p_{2}$ is invoked, op 1 is executed before op $p_{2}$ in each process $p_{i}$ 's local copy local_obj $j_{i}$.

Proof. By Lemma C.11, since every mutator costs at least $\epsilon$ time to respond, $o p_{2}$ is invoked more than $\epsilon$ time later than $o p_{1}$ is invoked and so the timestamp of $o p_{2}$ must be larger than that of $o p_{1}$. By Lemma C.10, since $o p_{2}$ has a larger timestamp than that of $o p_{1}$ and all the mutators are executed with timestamps ascending in each local copy, $o p_{1}$ is executed before $o p_{2}$ on $l o c a l_{-} o b j_{i}$.

ii. Pure Accessor after Pure Accessor

Lemma C.13 If a pure accessor aop $p_{1}$ responds in process $p_{i}$ before another pure accessor aop ${ }_{2}$ is invoked in process $p_{j}$, all the mutators executed on local_obj $j_{i}$ before $a_{0} p_{1}$ returns are executed on local_obj $j_{j}$ before aop 2 returns.

Proof. By Lemma C.7, the time between invocation of a pure accessor and its response is $d+\epsilon-X \geq u$ (recall that $0 \leq X \leq d+\epsilon-u$ ).

Assume in contradiction that there exists a mutator $\langle o p$, arg,ts $\rangle$ which is executed on $l o c a l_{-} o b j_{i}$ before $a o p_{1}$ responds but executed on $l o c a l \_o b j_{j}$ after $a o p_{2}$ responds. Because all the pure accessors use the same $X$ value, if $a p_{2}$ is invoked after 
$a o p_{1}$ returns, it must be invoked at least $d+\epsilon-X \geq u>\epsilon$ time later than $a p_{1}$ is invoked (recall we have optimal $\epsilon$, so $u>\epsilon$ ) and so it has a larger timestamp than $a o p_{1}$. By Lemma C.9 and Observation C.4, since op is executed on local_obj $j_{i}$ before aop responds, ts must be larger than the timestamp of $a o p_{2}$, which is larger than the timestamp of $a o p_{1}$

Now we have $t s$ is larger than the timestamp of $a o p_{1}$ and $o p$ is executed before $a o p_{1}$ returns. So there must exist an operation $\left\langle o p^{\prime}, a r g^{\prime}, t s^{\prime}\right\rangle$ with $t s^{\prime} \geq t s$ such that expire_timer $\left(\left\langle o p^{\prime}, a r g^{\prime}, t s^{\prime}\right\rangle\right.$, execute) occurs before $a p_{1}$ returns. Suppose $o p^{\prime}$ is invoked at real time $t$. By Observation C.1, in process $p_{i}$, the earliest time that set_timer $\left(u+\epsilon,\left\langle o p^{\prime}, a r g^{\prime}, t s^{\prime}\right\rangle\right.$, execute) occurs is $t+d-u$ and the earliest time expire_timer $\left(\left\langle o p^{\prime}, a r g^{\prime}, t s^{\prime}\right\rangle\right.$, execute $)$ occurs is $t+d+\epsilon$. So aop 1 returns after $t+d+\epsilon$, which means $a o p_{2}$ returns after $t+d+\epsilon+u$. By Observation C.1, in process $p_{j}$, the latest time when set_timer $\left(\left\langle o p^{\prime}, a r g^{\prime}, t s^{\prime}\right\rangle\right.$, execute) occurs is $t+d$, and the latest time when expire_timer $\left(\left\langle o p^{\prime}, a r g^{\prime}, t s^{\prime}\right\rangle\right.$, execute) occurs is $t+d+u+\epsilon$, which is before $a o p_{2}$ responds. Because $t s^{\prime} \geq t s$, the criteria to execute op on $l o c a l \_o b j_{j}$ is reached and so $o p$ is executed on $l o c a l \_o b j_{j}$ before $a o p_{2}$ returns, contradicting the assumption about the existence of $o p$.

iii. Pure Accessor after Mutator

Lemma C.14 If a pure accessor aop is invoked by $p_{i}$ after a mutator op responds, $p_{i}$ executes op on local_obji before aop responds.

Proof. We prove with two cases as below:

- $o p$ is a pure mutator.

Assume op is invoked at real time $t$ (local time T). Because op costs $\epsilon+X$ time, aop must be invoked after $t+\epsilon+X$. Because the local clock skew is 
bounded by $\epsilon$, at any real time after $t+\epsilon+X$, the local time of $p_{i}$ is larger than $(T-\epsilon)+\epsilon+X=T+X$. Then the timestamp for aop must be larger than $T+X-X=T$. By Observation C.4 and Lemma C.9, op is executed by $p_{i}$ before aop returns.

- op is not a pure mutator.

If $o p$ is not a pure mutator, the response time depends on the operations in the priority queue To_Execute of its invoking process.

Assume op is invoked by process $p_{j}$ and responds at real time $t_{r e s}$. There must be a mutator $\left\langle o p^{\prime}, a r g^{\prime}, t s^{\prime}\right\rangle$ in To_Execute $_{j}$ such that expire_timer $\left(\left\langle o p^{\prime}, a r g^{\prime}, t s^{\prime}\right\rangle\right.$, execute) occurs at $t_{r e s}$ and $t s^{\prime} \geq t s$. By the message delay uncertainty $u$, in process $p_{i}$, at real time $t_{r e s}+u$, expire_timer $\left(\left\langle o p^{\prime}, a r g^{\prime}, t s^{\prime}\right\rangle\right.$, execute $)$ must have occurred, and so op is executed on local_obj $_{i}$ no later than $t_{\text {res }}+u$. Since $0 \leq X \leq d+\epsilon-u$, aop responds after $t_{\text {res }}+d+\epsilon-X \geq t_{\text {res }}+u$. So $p_{i}$ executes op on $l o c a l_{-} o b j_{i}$ before aop responds.

iv. Mutator after Pure Accessor

Lemma C.15 If a mutator op is invoked after a pure accessor aop returns, aop's invoking process will execute op on its local copy after aop returns.

Proof. op can only be executed on the processes' copies after it is invoked and so after the return of aop. 


\section{c. Step III}

Now we construct a permutation $\pi$ of any run and then prove $\pi$ is legal and respects the real time sequence of non-overlapping operations. By Lemma C.10, we already proved that every process executes the mutators in the same order, with timestamps ascending. We arrange all the mutators with their timestamps ascending in $\pi$. Then we insert each pure accessor aop into the permutation after the latest mutator which is executed on the local copy in aop's invoking process before aop responds and before the following mutators.

If there are more than one pure accessors inserted between the mutator $o p_{1}$ and the mutator $o p_{2}$, order them by the real times of their invocations, breaking ties with process ids.

Lemma C.16 If an operation op $p_{1}$ responds before another operation op $p_{2}$ is invoked, $o p_{1}$ appears before $\mathrm{op}_{2}$ in $\pi$.

ProOF. We prove with 4 cases as below:

- $o p_{1}$ and $o p_{2}$ are both mutators: By Lemma C.11, op $p_{1}$ responds at least $\epsilon$ time after its invocation. Since $o p_{2}$ is invoked after $o p_{1}$ responds, it is invoked more than $\epsilon$ time after $o p_{1}$ is invoked. So $o p_{2}$ has a larger timestamp than $o p_{1}$ and is after $o p_{1}$ in $\pi$.

- $o p_{1}$ and $o p_{2}$ are both pure accessors: By Lemma C.13, all the mutators executed on the local copy of $o p_{1}$ 's invoking process before $o p_{1}$ responds are executed on the local copy of $o p_{2}$ 's invoking process before $o p_{2}$ responds. Suppose in the construction of $\pi, o p_{1}$ is inserted between the mutators $o p$ and $o p^{\prime}$. Then $o p$ is executed on the local copy of $o p_{1}$ 's invoking process before $o p_{1}$ responds, so it 
is also executed on the local copy of $o p_{2}$ 's invoking process before $o p_{2}$ responds. Therefore $o p_{2}$ must be inserted into a position after $o p$.

- $o p_{2}$ is also inserted between $o p$ and $o p^{\prime}$. Under this condition, since $o p_{1}$ and $o p_{2}$ are ordered according to the real times of their invocations, $o p_{1}$ is before $o p_{2}$.

- $o p_{2}$ is inserted somewhere after $o p^{\prime}$. Then it is trivial $o p_{2}$ is after $o p_{1}$ in $\pi$.

- $o p_{1}$ is a mutator and $o p_{2}$ is a pure accessor: By Lemma C.14, since $o p_{1}$ responds before $o p_{2}$ is invoked, $o p_{1}$ is executed on the local copy of $o p_{2}$ 's invoking process before $o p_{2}$ responds. Then by the construction of $\pi, o p_{2}$ is inserted at some position after $o p_{1}$.

- $o p_{1}$ is a pure accessor and $o p_{2}$ is a mutator: In the construction of $\pi, o p_{1}$ must be inserted somewhere before $o p_{2}$, otherwise $o p_{2}$ is executed on the local copy of $o p_{1}$ 's invoking process before $o p_{1}$ responds, contradicting Lemma C.15.

Lemma C.17 $\pi$ is legal.

ProOF. We prove by induction on the length of $\pi$.

Base: $\pi$ is an empty sequence. There is no operation, so $\pi$ is vacuously legal.

Induction step: Suppose $\pi=\pi^{\prime} \circ o p$, with the assumption $\pi^{\prime}$ is legal, we show $\pi$ is legal. It is proved in two cases as below:

- op $\in O O P \cup A O P$ : By the construction of $\pi$ (mutators are ordered with timestamps ascending and how pure accessors are inserted) and Lemma C.10, before $o p$ is executed on the local copy of its invoking process, op's invoking process has 
executed all the mutators in $\pi$ based on their timestamps ascending sequentially on its local copy, which is exactly the same as the sequence in $\pi$. Since the pure accessors in $\pi$ do not modify the object, it does not make any difference to the execution of $o p$ if $o p$ 's invoking process executes them on its local copy or not. Therefore the execution of op by op's invoking process on its local copy must return a value such that $\pi \circ$ op is legal.

- $o p \in M O P:$ op is always legal because it does not return any information about the object (refer to Definition D.3).

Lemma C.16 and Lemma C.17 prove:

Theorem C.18 Algorithm 1 is a correct implementation of a linearizable object with an arbitrary data type.

D. Analysis of Implementations

Theorem D.1 For all mop $\in$ MOP and aop $\in A O P,|m o p|+|a o p|=d+2 \epsilon$.

When $X$ is 0 , the time cost for a pure mutator is $\epsilon$.

With optimal $\epsilon$, the time cost is $(1-1 / n) u$, matching the time lower bound (refer to Theorem D.1) in the condition $k=n$. Therefore $(1-1 / n) u$ is a tight time bound for any pure mutator that is eventually non-self-last-permuting, such as write on a $\mathrm{read} /$ write register, push on a stack and enqueue on a queue.

When $X$ is $d+\epsilon-u$, the time cost for a pure accessor is $u$, leaving a gap $u / 2$ to the lower bound [1].

Theorem D.2 For all oop $\in O O P, \mid$ oop $\mid \leq d+\epsilon$. 
When $\epsilon \leq u$ and $\epsilon \leq d / 3$, this upper bound matches the lower bound for immediately non-self-commuting operations (refer to Theorem C.1). 


\section{CHAPTER VI}

\section{TIME BOUNDS FOR SPECIFIC SHARED OBJECTS}

In this chapter, we summarize the new lower bounds and upper bounds for specific objects, and compare them with previous results.

\section{A. Operations on Read/Write/Read-Modify-Write Registers}

There are three operations on a Read/Write/Read-Modify-Write Register: read, write and read-modify-write. Read is a pure accessor. Write is a pure mutator. Readmodify-write is an immediately non-self-commuting operation. With the implementation in Chapter V (Algorithm 1), the read operation costs $d+\epsilon-X$ time and the write operation costs $\epsilon+X$, where $X$ is a variable within $[0, d+\epsilon-u]$. Then the write operation costs $\epsilon$ when $X$ is set to 0 and so the write operation costs $(1-1 / n) u$ when $\epsilon$ is optimal, which matches the time lower bound for pure mutators (Theorem D.1). Besides, the read-modify-write operation $\operatorname{costs} d+\epsilon$ in the implementation, partially matching the time lower bound for immediately non-self-commuting operations (Theorem C.1). The most significant gap between time upper bound and lower bound is the time cost for $\mid$ write $|+|$ read $\mid$. The gap is $2 \epsilon$, which actually depends on the message delay uncertainty. The time bounds are shown as Table I.

\section{B. Operations on Queues and Stacks}

The operations on a queue and those on a stack are very similar. Peek is a pure accessor. Enqueue and push are pure mutators. Dequeue and pop are immediately non-self-commuting operations. The time complexities of enqueue and push operations are exactly the same as for the write operation on a register, whose tight time 
Table I. Summary of Operation Time Bounds on Read/Write/Read-Modify-Write

\begin{tabular}{|c|c|c|c|}
\hline \multicolumn{1}{|c|}{ Register } \\
\hline operations & Previous Lower Bound & Lower Bound & Upper Bound \\
\hline read-modify-write & $d[3]$ & $d+\min \{\epsilon, u, d / 3\}$ & $d+\epsilon$ \\
write & $u / 2[1]$ & $(1-1 / n) u$ & $\epsilon$ \\
read & $u / 2[3]$ & - & $u$ \\
write + read & $d[5]$ & $d$ & $d+2 \epsilon$ \\
\hline
\end{tabular}

bound is $(1-1 / n) u$. The time complexity of dequeue and pop operations are exactly the same as for the read-modify-write on a register, whose tight time bound is $d+\epsilon$ with $\epsilon \leq u$ and $\epsilon \leq d / 3$. The difference from the analysis on a read/write/readmodify-write register is the time complexity of $(|p u s h|$ or $\mid$ enqueue $\mid)+\mid$ peek $\mid$. Because the push operation and the enqueue operation do not overwrite the whole state of the object, the lower bound for $(\mid$ push $\mid$ or $\mid$ enqueue $\mid)+\mid$ peek $\mid$ is $d+\epsilon$ while the lower bound for $\mid$ write $|+|$ read $\mid$ is $d$. The time bounds are shown as Table II and Table III.

Table II. Summary of Operation Time Bounds on Queue

\begin{tabular}{|c|c|c|c|}
\hline operations & Previous Lower Bound & Lower Bound & Upper Bound \\
\hline enqueue & $u / 2[1]$ & $(1-1 / n) u$ & $\epsilon$ \\
dequeue & $d[3]$ & $d+\min \{\epsilon, u, d / 3\}$ & $d+\epsilon$ \\
enqueue + peek & $d[3]$ & $d+\min \{\epsilon, u, d / 3\}$ & $d+2 \epsilon$ \\
\hline
\end{tabular}

\section{Operations on Trees}

We consider four operations on a rooted tree: insert, delete, search and depth. Insert and delete are pure mutators. Search and depth are pure accessor. There is no 
Table III. Summary of Operation Time Bounds on Stack

\begin{tabular}{|c|c|c|c|}
\hline operations & Previous Lower Bound & Lower Bound & Upper Bound \\
\hline push & $u / 2[1]$ & $(1-1 / n) u$ & $\epsilon$ \\
pop & $d[3]$ & $d+\min \{\epsilon, u, d / 3\}$ & $d+\epsilon$ \\
push + peek & $d[3]$ & $d+\min \{\epsilon, u, d / 3\}$ & $d+2 \epsilon$ \\
\hline
\end{tabular}

operation which is both mutator and accessor. The time bounds result is summarized as Table IV:

Table IV. Conclusions of Operation Time Bounds on Tree

\begin{tabular}{|c|c|c|c|}
\hline operations & Previous Lower Bound & Lower Bound & Upper Bound \\
\hline insert & $u / 2[3]$ & $(1-1 / n) u$ & $\epsilon$ \\
delete & $u / 2[3]$ & $(1-1 / n) u$ & $\epsilon$ \\
insert + depth & $d[3]$ & $d+\min \{\epsilon, u, d / 3\}$ & $d+2 \epsilon$ \\
delete + depth & $d[3]$ & $d+\min \{\epsilon, u, d / 3\}$ & $d+2 \epsilon$ \\
\hline
\end{tabular}




\section{CHAPTER VII}

\section{CONCLUSION}

In this work, we studied the properties of operations on an arbitrary object with linearizability. Then we showed the time lower bounds and upper bounds for three categories of operation types: immediately non-self-commuting operations, eventually non-self-commuting operations and immediately non-commuting pair of operations. In each category, we present our results on time bounds and discuss the remaining future work:

The time lower bound for immediately non-self-commuting operation types is $d+\min \{\epsilon, u, d / 3\}$ and the time bound is tight if $\epsilon \leq d / 3$ ( $d$ is message delay upper bound, $u$ is message delay uncertainty, $\epsilon$ is local clock skew upper bound). The tight time bound for the case $\epsilon>d / 3$ is still unknown. Considering the optimal $\epsilon=(1-1 / n) u$, if we have $u \leq d / 3$, it is easy to get $\epsilon \leq d / 3$. So the challenge comes from the message uncertainty $u$, if it is larger than $d / 3$. From an intuitive view, with a larger message uncertainty, each process can get less information (about when the message is sent) from time when the message is received, and therefore it is harder to decide the sequence of concurrent operations.

For the operation types of which there exist $k$ instances, such that different permutations of them are not equivalent, the lower bound is $(1-1 / k) u$. For eventually non-self-last-permuting operation types, $k=n$, and so the lower bound is $(1-1 / n) u$ and it is a tight time bound when the local clock skew upper bound is optimal. Although this result can be applied to many commonly used objects such as registers, queues and stacks, it remains an open question about the tight time lower bound for operation types which are eventually non-self-commuting but not eventually non-selflast-permuting. Since there are $k$ ! possible permutations for $k$ concurrent operations, 
and we have studied the case where two permutations have different last operations, it is a challenge to study all the other cases.

The time lower bound for a pair of the non-commuting operation types $O P$ and $A O P$ depends on the properties of $O P$. If $O P$ is eventually self-commuting, such as insert and delete on a set, or if $O P$ is eventually non-self-commuting but overwriting, such as the write operation on a register, the lower bound is $d$, there remains a gap of $2 \epsilon$ with the upper bound, according to our implementation where $|M O P|+|A O P|=$ $d+2 \epsilon$. If $O P$ is eventually non-self-commuting and non-overwriting, such as enqueue on a queue and push on a stack, the lower bound is $d+\epsilon$. We narrowed the gap between upper and lower bound, but still leave a gap of $\epsilon$.

In this work, we assumed bounded local clock skew without time drift. The partially synchronous model with bounded clock skew and bounded time drift needs to be explored in the future works. We may also consider different types of failures in message passing systems. 


\section{REFERENCES}

[1] H. Attiya and J.L. Welch, "Sequential consistency versus linearizability," $A C M$ Trans. Computer Syst., vol. 12, pp. 91-122, May 1994.

[2] M.P. Herlihy and J.M. Wing, "Linearizability: A correctness condition for concurrent objects," ACM Trans. Program. Languages Syst., vol. 12, pp. 463-492, July 1990.

[3] M.J. Kosa, "Time bounds for strong and hybrid consistency for arbitrary abstract data types," Chicago Journal of Theoretical Computer Science, 1999.

[4] L. Lamport, "On Interprocess Communication," Distributed Computing, vol. 1, no. 2, pp. 86-101, 1986.

[5] R.J. Lipton and J.S. Sandberg, "PRAM: A scalable shared memory," Technique Report CS-TR-180-88, Princeton Univ., Dept. of Computer Science, 1988.

[6] J. Lundelius and N. Lynch, "An upper and lower bound for clock synchronization," Information and Control, vol. 62, no. 2-3, pp. 190-204, 1984.

[7] M. Mavronicolas and D. Roth, "Linearizable read/write objects," in Theoretical Computer Science, vol. 220, no. 1, pp. 267-319, June 1999.

[8] W.E. Weihl, "Commutativity-based concurrency control for abstract data types," in IEEE Trans. Computers, vol. 37, no. 12, pp. 1488-1505, 1988. 


\section{VITA}

Name: JIAQI (Erica) WANG

E-mail: ericaqi@cse.tamu.edu Mobile: (979) 393-8528

Address: Department of Computer Science and Engineering

Texas A\&M University

TAMU 3112, c/o Jennifer Welch

College Station, TX 77843-3112

EDUCATION

Aug 2008-Dec 2011 in Texas A\&M University, College Station, Texas

Master of Science in Computer Science and Engineering

Sep 2001-Jun 2005 in Nanjing University, Nanjing, China

Bachelor of Science in Computer Science

COURSE PROJECT EXPERIENCE

Aug 2008-Present Computer Science $\&$ Engineering, Texas A\&M University

Tiny-SQL Interpreter, Container Loading Recommendation, SOM Clustering, Par-

allel Hierarchical Clustering, Trust Propagation with Controversial User and Online Mini Texas Hold'em

RESEARCH EXPERIENCE

Aug 2008-May 2009 in Industrial Engineering, Texas AEM University

Project: Intelligent Tutoring System for Automatic Assembly System

Jan 2010-Present in Computer Science EEngineering, Texas A\&M University

Project: Distributed Algorithms Design and Analysis

PUBLICATION

Brief Announcement: Time Bounds for Shared Objects in Partially Synchronous Systems, PODC'11 